\title{
Bignoniaceae in the Raso da Catarina Ecoregion, Bahia, Brazil
}

\author{
Luiza Regina Silva ${ }^{1 *}$, Milene Maria da Silva-Castro ${ }^{2} \&$ Adilva de Souza Conceição $^{I}(\mathbb{C}$ \\ ${ }^{1}$ Universidade do Estado da Bahia, Programa de Pós-Graduação em Biodiversidade Vegetal, Herbário \\ HUNEB (Coleção Paulo Afonso), Campus VIII, Departamento de Educação, Rua do Gangorra, 503, CHESF, \\ 48.608-240, Paulo Afonso, BA, Brasil \\ ${ }^{2}$ Universidade Estadual do Sudoeste da Bahia, Departamento de Ciências Biológicas, Campus de Jequié, \\ Rua José Moreira sobrinho s/n, 45200-000, Jequié, BA, Brasil \\ *Corresponding author: Luiza Regina Silva, e-mail: lregina.bio@gmail.com
}

SILVA, L.R., CASTRO, M.M.S., CONCEIÇÃO, A.S. Bignoniaceae in the Raso da Catarina Ecoregion, Bahia, Brazil. Biota Neotropica. 18(4): e20170466. http://dx.doi.org/10.1590/1676-0611-BN-2017-0466

\begin{abstract}
This work presents a floristic survey of the family Bignoniaceae in the Raso da Catarina Ecoregion, Bahia, Brazil, considered as the main family of the Brazilian forests lianas. The samples analyzed were collected from September 2012 to May 2014. The analyses were supplemented with botanical collections kept in the herbaria: ALCB, HRB, HUEFS, IPA and PEUFR. The identifications were made based mainly on specialized bibliographies, protologues, photos of type collections and consulting of the collections in the herbaria that were visited. 11 genera and 20 species of the family were recorded. Fridericia Mart. and Handroanthus Mattos were the most representative genera ( $5 \mathrm{spp}$.), followed by Jacaranda Juss ( $2 \mathrm{spp}$.). The other genera presented only one species each. The most representative species in the area were: Anemopaegma laeve DC., Fridericia erubescens (DC.) L.G.Lohmann, Jacaranda jasminoides (Thunb.) Sandwith. and Tabebuia aurea (Manso) Benth. \& Hook f. ex Moore. Among the species registered one is new record for the Caatinga biome, ten are endemic to Brazil and three exclusive of the Caatinga. The taxonomic treatment includes a key for the identification, descriptions, illustrations, photos, data of the geographical distribution, economic potential and reproductive phenology and comments about the species.

Keywords: semiarid, caatinga, taxonomy, diversity.
\end{abstract}

\section{Bignoniaceae na Ecorregião Raso da Catarina, Bahia, Brasil}

Resumo: Este trabalho apresenta o levantamento florístico da família Bignoniaceae na Ecorregião Raso da Catarina, Bahia, Brasil, considerada uma das principais famílias de lianas das florestas brasileiras. Os espécimes analisados foram coletados no período de setembro 2012 a maio 2014. As análises foram complementadas por coleções botânicas depositadas nos herbários: ALCB, HRB, HUEFS, IPA e PEUFR. As identificações foram realizadas com base, principalmente, em bibliografias especializadas, protólogos, imagens de coleções-tipo e consultas às coleções dos herbários visitados. Foram catalogados 11 gêneros e 20 espécies. Fridericia Mart. e Handroanthus Mattos foram os gêneros mais representativos (5spp.), seguido de Jacaranda Juss (2spp.). Os demais gêneros estão representados por uma espécie cada um. As espécies mais representativas na área foram: Anemopaegma laeve DC., Fridericia erubescens (DC.) L.G.Lohmann, Jacaranda jasminoides (Thunb.) Sandwith. e Tabebuia aurea (Manso) Benth. \& Hook f. ex Moore. Dentre as espécies catalogadas, uma é nova ocorrência para o bioma Caatinga, dez são endêmicas do Brasil, sendo três exclusivas da Caatinga. O tratamento taxonômico inclui uma chave de identificação, descrições, ilustrações, fotos, dados de distribuição geográfica, potencial econômico, fenologia reprodutiva e comentários sobre as espécies.

Palavras-chave: semiárido, caatinga, taxonomia, diversidade. 


\section{Introduction}

Bignoniaceae with 827 species allocated in 82 genera (Lohmann \& Ulloa 2007), has a predominantly Neotropical distribution with its center of diversity in Brazil (Gentry 1980). The species occur in a variety of environments, from open savannas to humid and perennial forests, and represent the main family of lianas in Brazilian forests (Silva \& Queiroz 2003), where 413 species and 33 genera are recorded (Lohmann 2015).

The family is included in the order Lamiales (APG IV 2016) based on molecular phylogenetic studies. With the exclusion of the tribe Schlegelieae and the genus Paulownia Siebold \& Zucc., which were included in the families Schlegeliaceae and Paulowniaceae, respectively, Bignoniaceae is considered monophyletic, with the synapomorphies of: absence of endosperm, composite leaves, and seeds with hyaline wings surrounding the embryo (Spangler \& Olmstead 1999, Olmstead et al. 2009).

After studies involving tribal and intergeneric delimitations, seven clades can be recognized for the family: Bignonieae, Catalpeae, Oroxyleae, Tecomeae, Tourrettieae, Jacarandeae and Crescentiina. This last group is inserted the clade informally denominated of Tabebuia Alliance, a Neotropical lineage formed by the tribe Crescentieae and by genera segregated from the tribe Tecomeae (Grose \& Olmstead 2007, Olmstead et al. 2009). Among these groups, we highlight Bignonieae as the largest tribe of Bignoniaceae, and which underwent taxonomic rearrangements after molecular phylogenetic studies resulting in the proposal of synonyms, new combinations and recircumscriptions of genera. Currently it includes 393 species and 21 genera (Lohmann \& Taylor 2014), and is the most diverse and abundant group of lianas in tropical forests of the New World (Gentry 1986). The tribe constitutes a monophyletic lineage strongly supported by molecular and morphological data (Spangler \& Olmstead 1999, Olmstead et al. 2009).

In addition to its great ecological importance, the family also possesses economic value expressed mainly by species with wood potential. Additionally, trees are ornamental when flowering, and are popular species for use in landscaping in Brazil, while some are still used to cover gates and fences in reforestation areas or in popular medicine (Gentry 1992a, Lorenzi 2008, 2013).

The Raso da Catarina Ecoregion (RCE) is one of the eight Ecoregions the Caatinga biome (Velloso et al. 2002). This biome has high rate of endemism and diversity, but is probably the most undervalued and poorly known botanically biome (Giulietti et al. 2002). In addition, it also has the lowest number of conservation units and is one of the least protected in Brazil (Leal et al. 2005). The most recent contributions about flora of Raso da Catarina Ecoregion are generally local floristic surveys of groups in specific areas, such as the works conducted by Melo \& Andrade (2007); Melo et al. (2010); Brito (2012); Lopes (2012); Dourado et al. (2013); Varjão et al. (2013); Vieira et al. (2013); Silva et al. (2016); Lima \& Conceição (2016); Santos et al. (2016) and; Correia \& Conceição (2017a, 2017b), with few floristic surveys covering all areas of the Ecoregion, such as Silva (2014); Lima (2015); Vieira et al. (2015); Amorim \& Bautista (2016) and; Santos (2016).

Important studies on the group in the Northeast Region of Brazil have been performed, however, studies on taxa of Bignoniaceae in areas of Caatinga in Bahia, including identification keys and descriptions are few. Given the significant rate of endemism and diversity for the Caatinga biome, and the limited number of surveys for the family therein, this study aims to contribute to a better understand of the family in the Raso da Catarina Ecoregion (Bahia portion), in order to contribute to the knowledge of the local flora and to provide support for the development of management plans for the conservation units (CUs) of this ecoregion.

\section{Material and Methods}

The Raso da Catarina Ecoregion comprises $30.800 \mathrm{~km}^{2}$ and is one of the eight Ecoregions recognized for the Caatinga. In the North-south direction it is narrow and elongated. In the North, West and East it is limited to the southern hinterland depression. The northeastern portion has limits with the Borborema Plateau and the southern part of the Bahia hinterland, in the Zona da Mata. The Ecoregion is a basin with soils that are very sandy, deep and little fertile. Its relief is very flat, but with canyons in the western part (formed by sandstone outcrops). The altitudes above sea level vary from 400 to $600 \mathrm{~m}$ in the southern part (Bahia) and from 350 to $700 \mathrm{~m}$ in the northern part (Jatobá basin, Pernambuco). In the southern part (Bahia) most of the soils are composed of sand (deep, excessively drained, acid and very low fertility) and oxisol (deep, well drained, acid and low fertility) whereas in the northern part (Pernambuco) sands soils prevail. There exists little surface water in the region except in the areas of the canyons. The predominant vegetation is the sandy, bushy Caatinga, very dense and less thorny than the Caatinga of crystalline soils (Velloso et al. 2002).

The climate of the Ecoregion is semiarid with average rainfalls of $650 \mathrm{~mm} /$ year in the southern part (Bahia) and rainy season from December to July. In the northern part (Pernambuco) the climate is drier with average rainfalls of $450 \mathrm{~mm} /$ year and the rainy period is from January to April. The ambient temperatures are very high with large differences of temperature between day and night. The soils are generally sandy deep and very fertile relief plan with sandstone formations and the predominant vegetation is the sandy, bushy Caatinga, very dense (Velloso et al. 2002).

The municipalities in this Ecoregion in the state of Bahia are: Canudos, Chorrochó, Cícero Dantas, Euclides da Cunha, Glória, Jeremoabo, Macururé, Paulo Afonso, Rodelas, Santa Brígida and Uauá. The Ecoregion includes six units of conservation, five being located in its southern part in the state of Bahia: Ecological Station Raso da Catarina, Environmental Protection Area (EPA) Serra Branca, State Park of Canudos, Biological Station of Canudos, RPPN Farm Flor de Lis and Biological Reserve of Serra Negra, located in the northern part, in the state of Pernambuco (Velloso et al. 2002, Szabo et al. 2007).

The expeditions concentrated in the conservation units (Figure 1) located in the southern part of the Ecoregion, portion Bahia; EPA Serra Branca $\left(09^{\circ} 53^{\prime} 15.5^{\prime \prime}\right.$ to $09^{\circ} 44^{\prime} 34.6^{\prime \prime} \mathrm{S}$ and $38^{\circ} 49^{\prime} 36.1^{\prime \prime}$ to $\left.38^{\circ} 52^{\prime} 20.4^{\prime \prime} \mathrm{W}\right)$; Biological Station of Canudos-BSC (09 $55^{\prime} 58.6^{\prime \prime}$ to $09^{\circ} 58^{\prime} 25.2^{\prime \prime} \mathrm{S}$ and $38^{\circ} 57^{\prime} 32.2^{\prime \prime}$ to $\left.39^{\circ} 01^{\prime} 38.5^{\prime \prime} \mathrm{W}\right)$; Ecological Station Raso da Catarina-ESRC (09³3' $13^{\prime \prime}$ to $09^{\circ} 54^{\prime} 30^{\prime \prime} \mathrm{S}$ and $38^{\circ} 29^{\prime} 20^{\prime \prime}$ to $\left.38^{\circ} 44^{\prime} 00^{\prime \prime} \mathrm{W}\right)$; State Park of Canudos-SPC (09 $56^{\prime} 19.7^{\prime \prime}$ to $09^{\circ} 54^{\prime} 32.4^{\prime \prime}$ $\mathrm{S}$ and $39^{\circ} 06^{\prime} 13.3^{\prime \prime}$ to $39^{\circ} 04^{\prime} 20.5^{\prime \prime} \mathrm{W}$ ) and RPPN Farm Flor de Lis (1050'14.1" to $10^{\circ} 50^{\prime} 26.4^{\prime \prime} \mathrm{S}$ and $38^{\circ} 31^{\prime} 46.4$ ' to $38^{\circ} 31^{\prime} 34.7^{\prime \prime} \mathrm{W}$ ) (Vieira et al. 2015).

The study was based on fieldwork carried out in the period between September 2012 and May 2014, besides information complemented by the analysis of specimens deposited in the herbaria: ALCB, HRB, HUEFS, IPA and PEUFR, acronyms according to Thiers (2018) 


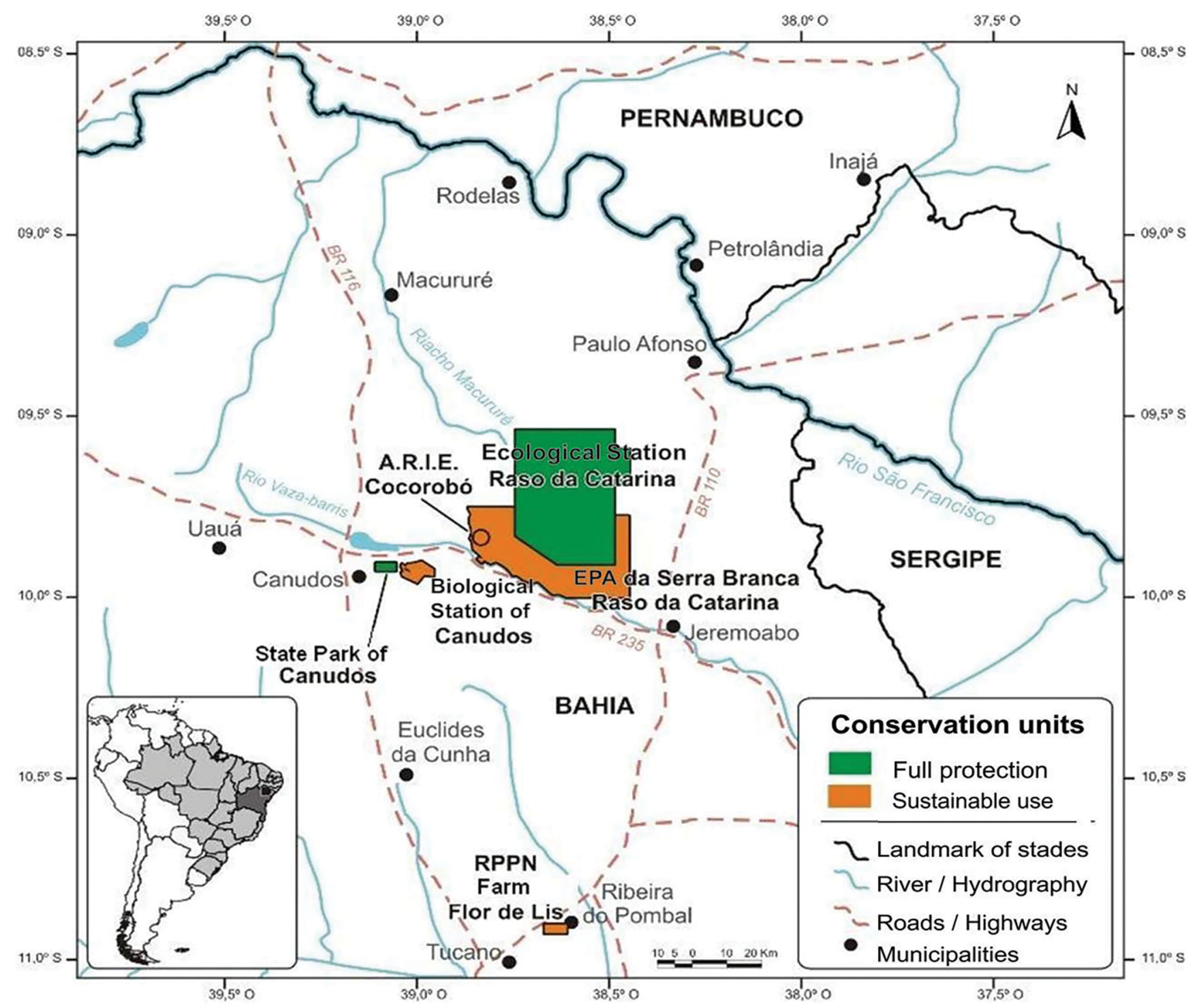

Figure 1. Location of conservation units of the Ecoregion Raso da Catarina, state of Bahia, Brazil (Varjão et al. 2013, modified).

(continuously updated; Appendix 1). The field collections and observations were performed during random walks exploring most of the study area. Herborization and material processing followed the methodology by Fosberg \& Sachet (1965) and Mori et al. (1989), where fertile material was collected with flowers and/or fruit. Observations were made about the distribution of the species and the type of soil (Sampaio 1995, Tricart 1972). The specimens were deposited in the herbarium HUNEB -Paulo Afonso Collection and the duplicates will be sent to the main herbaria in the state of Bahia.

The identifications were made based mainly on specialized bibliographies (e.g., Woodson et al. (1973), Gentry (1982), Lohmann \& Pirani (1996), Rizzini et al. (1997), Silva \& Queiroz (2003), Scudeller (2004), Silva-Castro et al. (2007), Grose \& Olmstead (2007), Espírito Santo et al. (2012), Espírito Santo et al. (2013), Santos et al. (2013), Lohmann \& Taylor (2014), protologues, photos of type collections and consulting of the collections in the herbaria that were visited. For the taxonomic descriptions, the terminologies proposed by Gonçalves \& Lorenzi (2011), Harris \& Harris (2001) and Radford et al. (1974) were adopted. The taxonomic treatment includes a key for the identification of taxa, descriptions, illustrations, and data of the geographical distribution and reproductive phenology of the species.

\section{Results and Discussion}

Bignoniaceae Juss., Gen. Pl. [Jussieu]. 137. 1789.

Trees, shrubs or lianas. Branches commonly cylindrical to quadrangular or hexagonal. Leaves opposite, rarely verticillate or alternate, composite, 2-5-foliolate, ternate, pinnate, bipinnate or digitate, rarely simple, margins entire, crenate or serrate, venation pinnatinerved to palmatinerved; in lianas terminal leaflet can be substituted by tendril (simple, bifid, trifid or multifid). Inflorescences racemose or tirsoid, terminal, axillary or cauliflorous. Flowers generally large and showy, dichlamydeous, pentamerous, zygomorphic, bisexual. Calyx gamosepalous, campanulate, tubular, spathaceous or urceolate, apex spatulate, bilabiate, truncate or 2-5-lobed, aestivation imbricate, with or without patelliform nectaries. Corolla gamopetalous, tubularcampanulate, infundibuliform, campanulate or hypocrateriform, sometimes with bilabiate apex, aestivation imbricate, zygomorphic; 
stamens included or exserted, 4, rarely reduced to 2, didynamous, staminode reduced, glabro, rarely elongate and pilose; filaments adnate to the corolla at the base; anthers commonly divergent, generally glabrous, rarely pilose; pollen grains many, in monads, rarely in tetrads or polyads; nectariferous disk generally present; gynoecium bicarpellate, bilocular; ovary superior, multiovulate, placentation axial, stigma bilamelate, lobes sensitive (closing after contact with pollinator). Fruit capsule usually dehiscent, septifragal or loculicidal, rarely indehiscent; seeds usually winged, rarely without wings (Woodson et al. 1973, Gentry 1980, Lohmann 2004).

In the RCE (southern part) 11 genera and 20 species were catalogued for the family. Fridericia Mart. and Handroanthus Mattos with five species, were the most representative genus, followed by Jacaranda Juss., with two species. The other genera presented only one species each. The most representative species in the area were: Anemopaegma laeve DC., Fridericia erubescens (DC.) L.G.Lohmann, Jacaranda jasminoides (Thunb.) Sandwith. and Tabebuia aurea (Manso) Benth. $\&$ Hook f. ex Moore.

\section{Identification key for the species of Bignoniaceae in the RCE}

1. Trees or erect shrubs; without tendrils.

2. Leaves digitate; staminode $\leq 10 \mathrm{~mm}$, glabrous.

3. Calyx with nectaries; corolla magenta with yellow nectar guides on the fauces ......... 12. Handroanthus impetiginosus 3'. Calyx without nectaries; corolla yellow without nectar guides on the fauces or when present reddish or brown.

4. Leaflets glabrescent, abaxial surface glabrescent with tector and stellate trichomes restricted on the midrib; corolla $\geq 10 \mathrm{~cm}$........................ 11. Handroanthus grandiflorus 4'. Leaflets tomentose or lepidote; corolla $\leq 10 \mathrm{~cm}$.

5. Calyx bilabiate, lepidote ............ 20. Tabebuia aurea

5'. Calyx 3-5-lobed, with tector trichomes, dendroid or stellate.

6. Calyx green-ferruginous, densely vilose; ovary pilose. 7. Leaflets with abaxial surfaces whitish-cream

13. Handroanthus ochraceus

7'. Leaflets with abaxial surfaces cream-ferruginous .. 10. Handroanthus chrysotrichus

6'. Calyx green passing the yellow, glabrous to sparse pubescent, ovary glandular-verrucose

14. Handroanthus serratifolius

2'. Leaves pinnate and/or bipinnate; staminode $\geq 28 \mathrm{~mm}$, glandular-pubescent.

8. Leaves bipinnate; calyx deeply lobed; anthers 1-thecate; fruit woody with margins broadly undulating

15. Jacaranda brasiliana

8 '. Leaves pinnate at the base and bipinnate at the apex; calyx cupulate; anthers 2-thecate; fruit coriaceous with margins flat

16. Jacaranda jasminoides

1'. Lianas or scandent shrubs; with tendrils.

9. Nectariferous disk inconspicuous or absent.

10. Calyx campanulate; corolla magenta with white fauces; anthers and connective glabrous, ovary papillate

2. Bignonia convolvuloides

10'. Calyx tubular; corolla pink to red with yellow fauces; anthers, connective and ovary pilose 17. Lundia longa
9'. Nectariferous disk conspicuous.

11. Stamens exserted.

12. Tendrils trifid; corolla orange, glabrous; fruit linear, without wings 19. Pyrostegia venusta 12'. Tendrils simple; corolla red to orange passing to pink after pollination, pubescent; fruit oblong, with wavy wings

11'. Stamens included.

13. Tendrils trifid.

14. Tendrils with uncinate tips; calyx spathaceous, with a recurved apicule; corolla yellow; connective not elongated, glabrous 4. Dolichandra quadrivalvis 14'. Tendrils with tips not uncinate; calyx campanulate, truncate or 5-mucronate; corola purple; connective elongated, pubescent

18. Mansoa paganuccii 13'. Tendrils simple.

15. Prophylls of the axillary buds foliaceous; calyx with nectaries; ovary stipitate .......... 1. Anemopaegma laeve 15'. Prophylls of axillary buds minute; calyx without nectaries; ovary sessile.

16. Leaflets with margins crenate

8. Fridericia parviflora

16'. Leaflets with margins entire.

17. Calyx cuspidate; anthers forward-curved, slightly pubescent ....................... 3. Cuspidaria lateriflora 17'. Calyx no cuspidate; anthers straight, glabrous.

18. Plant densely pubescent; with interpetiolar glands conspicuous; corolla $\leq 2.5 \mathrm{~cm}$

9. Fridericia pubescens

18'. Plant glabrous or glabrescent; without interpetiolar glands; corolla $\geq 3 \mathrm{~cm}$.

19. Leaflets membranous; calyx campanulate; corolla tomentose, lilac with lobes passing to magenta, white fauces

5. Fridericia dichotoma

19'. Leaflets chartaceous; calyx slightly urceolate; corolla pubescent, white passing to cream, white to cream fauces

7. Fridericia limae

1. Anemopaegma laeve DC., Prodr. [A. P. de Candolle]. 9: 189. 1845. Figures $2 \mathrm{~A} ; 3 \mathrm{~A}-\mathrm{F}$

Liana; branchlets cylindrical, striated, without lenticels, pubescent, with interpetiolar glands. Prophylls of the axillary buds foliaceous ca. $1.5 \times 1.3 \mathrm{~cm}$, orbicular, pubescent, trichomes tector. Leaves 2-foliolate, petioles $2.5-3 \mathrm{~cm}$, petiolules ca. $0.4 \mathrm{~cm}$, both pubescent; leaflets $4.5-6 \times$ $2-2.5 \mathrm{~cm}$, concolorous, chartaceous or coriaceous, lanceolate to elliptic, apex acuminate to rounded, margins entire, base rounded, venation penninerved, both surfaces pubescent with tector trichomes; tendrils simple, with tips not uncinate. Inflorescence a thyrse, peduncles ca. 1 $\mathrm{cm}$, pedicel ca. $1 \mathrm{~cm}$, both pubescent, bracts and bracteoles caducous. Calyx ca. $1 \times 1 \mathrm{~cm}$, green, tubular to campanulate, truncate, without rifts, slightly pubescent, tector trichomes sparse, with nectaries; corolla 3-5 $\times$ ca. $1.5 \mathrm{~cm}$, tube and fauces yellow and lobes white, infundibuliform, slightly pubescent; stamens included, dorsal filaments ca. $2.3 \mathrm{~cm}$, ventral filaments ca. $1.5 \mathrm{~cm}$, anthers ca. $8 \mathrm{~mm}, 2$-thecate, glabrous, straight, 
connective not elongated, glabrous, staminode ca. $5 \mathrm{~mm}$, glabrous; nectariferous disk conspicuous; ovary ca. $5 \times 1 \mathrm{~mm}$, glabrous, stipitate, not ridged, style ca. $3.3 \mathrm{~cm}$, stigma ca. $3 \mathrm{~mm}$. Capsules $6-8 \times 4.5-5.5$ $\mathrm{cm}$, ovate to orbicular, enlarged and flattened, smooth, coriaceous, margins flat, without wings. Seeds $2.5 \times 3.3 \mathrm{~cm}$.

Material examined: BRAZIL. BAHIA: Canudos, Estação Biológica de Canudos, Saco do gavião, próximo a igrejinha, $09^{\circ} 57^{\prime} 68^{\prime \prime} \mathrm{S}$ $39^{\circ} 00^{\prime} 11^{\prime \prime} \mathrm{W}, 427$ m, 17.V.2012, fl., D.D. Vieira et al. 290 (HUNEB); Toca da Velha estrada em direção ao saco, 0957'53.6"S 38 $59^{\prime} 75.5^{\prime \prime} \mathrm{W}$ 510 m, 27.II.2012, fl., D.D. Vieira et al. 224 (HUNEB); Euclides da Cunha, Sucupira do galo, $10^{\circ} 21^{\prime} 01.1^{\prime \prime} \mathrm{S} 38^{\circ} 41^{\prime} 11.5^{\prime \prime} \mathrm{W}, 547 \mathrm{~m}, 16 . \mathrm{I} .2014$, fr., L.R. Silva et al. 269 (HUNEB); Glória (RPPN), Complexo Itaparica, 0903'08"S 57¹5'09" W, 325 m, 24.XI.2006, fl.; fr., Oliveira. M 2477 (PEUFR). Jeremoabo, APA Serra Branca, Fazenda Serra Branca, Baixa dos Mandacarus, 0952'27.2"S 38 40'02.6"W 443 m, 10.V.2013, fl., L.R. Silva et al. 167 (HUNEB); Baixa da forca, $10^{\circ} 00^{\prime} 04.6^{\prime \prime} \mathrm{S} 38^{\circ} 28^{\prime} 46.4^{\prime \prime} \mathrm{W}$, 562 m, 23.I.2013, fl.; fr., L.R. Silva et al. 107, 110 (HUNEB); Baixa da estrada que vai da Estação Ecológica Raso da Catarina aos Quelés, 09 $53^{\prime} 43.9^{\prime \prime} \mathrm{S} 33^{\circ} 30^{\prime} 34.6^{\prime \prime} \mathrm{W}, 548$ m, 27.I.2011, fl., R.R. Varjão et al. 19 (HUNEB); Baixa dos Quelés, 0958'59"S 38²6'24"W, 506 m, 17.X.2009, fl., E. Melo et al. 6690 (ALCB); Entrada da APA próximo à porteira, $09^{\circ} 57^{\prime} 30.1^{\prime \prime} \mathrm{S} 38^{\circ} 26^{\prime} 19.3^{\prime \prime} \mathrm{W}, 524 \mathrm{~m}, 28 . X I .2011$, fl.; fr., R.R. Varjão et al. 35 (HUNEB); Estrada em direção a ESEC, 0957'20.0"S $38^{\circ} 26^{\prime} 73.3^{\prime \prime} \mathrm{W}, 554 \mathrm{~m}, 22 . X .203$, fl., L.R. Silva et al. 230 (HUNEB); Estrada em direção ao povoado Quelés, $10^{\circ} 00^{\prime} 0.78^{\prime \prime} \mathrm{S} 38^{\circ} 23^{\prime} 70.6^{\prime \prime} \mathrm{W}$, 592 m, 19.XII.2012, fl., L.R. Silva et al. 74,75 (HUNEB); Estrada principal em direção a ESEC depois da casa de Sr. Antônio Roxinho, 0957'16.1"S 38²6'23.2"W, 505 m, 19.XII.2013, fl., L.R. Silva et al. 246 (HUNEB); Fazenda Serra Branca, próximo ao rio Vaza Barris, 0956'39.4"S 3842'17.2"W, 01.XI.2008, fr., M.V.V. Romão et al. 410 (HUNEB); Paulo Afonso, Estação Ecológica do Raso da Catarina, Brejo do Burgo, Serrota, s/c, 11.I.193, fl., F.P. Bandeira 135 (ALCB); Trilha antes da curva das Solanáceas, 0949'05.4"S 38²9'31.2"W, 695 m, 24.IX.2013, fl., L.R. Silva et al. 206 (HUNEB); Santa Brígida, $10^{\circ} 45^{\prime} \mathrm{S} 38^{\circ} 17^{\prime} \mathrm{W}, 26 . X .1981$, fl., R.P. Orlandi 554 (HRB); Rodelas, s/c, 3.VIII.1994, fr., M.C. Ferreira 607 (HRB).

Endemic species of Brazil, occurring in the states of Bahia, Ceará, Maranhão, Minas Gerais, Paraíba, Pernambuco and Piauí, in Caatinga and Cerrado environments (Lohmann 2015). It is very well distributed in the Ecoregion being one of the most common species, recorded to three conservation units: EBC, EPA Serra Branca and ESRC. It flowering and fruiting a good part of the year mainly between the months of October to February.

Anemopaegma laeve can be easily recognized by foliaceous prophylls, calyx with nectaries, corolla with tube and fauces yellow and lobes white, stipitate ovary and capsule flattened and enlarged, and ovate to orbicular.

\section{Bignonia convolvuloides (Bureau \& K. Schum.)}

L.G.Lohmann, Ann. Missouri Bot. Gard. 99: 418. 2014.

Figures 2B; 3G-K

Liana; branchlets cylindrical, striated, with lenticels, pubescent, without interpetiolar glands. Prophylls of the axillary buds bromeliadlike (minute and triangular), ca. $0.2 \times 0.1 \mathrm{~cm}$, glabrous. Leaves 2-3foliolate, petioles $2-2.5 \mathrm{~cm}$, pubescent, petiolules ca. $0.6 \mathrm{~cm}$, tomentose, leaflets $5.5-11 \times 3-6.5 \mathrm{~cm}$, concolorous, chartaceous to membranaceous, elliptic or oblong, apex acuminatte, rarely rounded, margins entire, base rounded to cuneate, venation penninerved, both surfaces with tector trichomes restricted on the midrib; tendrils simple, with tips not uncinate. Inflorescence a raceme, peduncles ca. $2.5 \mathrm{~cm}$, pilose, pedicel ca. $1.5 \mathrm{~cm}$, tomentose, bracts and bracteoles inconspicuous. Calyx ca. $0.4 \times 0.6 \mathrm{~cm}$, base green, apex magenta, campanulate, 5-lobed, apiculate, without rifts, glabrescent, without nectaries; corolla 6-7 $\times 2-2.5 \mathrm{~cm}$, magenta with white fauces, infundibuliform, glabrous; stamens included, dorsal filaments ca. $1.5 \mathrm{~cm}$, ventral filaments ca. 1 $\mathrm{cm}$, anthers ca. $10 \mathrm{~mm}, 2-$ thecate, glabrous, straight, connective not elongated, glabrous, staminode ca. $6 \mathrm{~mm}$, glabrous; nectariferous disk inconspicuous, ovary ca. $4 \times 1 \mathrm{~mm}$, papillate, sessile, not ridged, style ca. $1.7 \mathrm{~cm}$, stigma ca. $3 \mathrm{~mm}$. Fruits and seeds not seen.

Material examined: BRAZIL. BAHIA: Glória, Povoado Retiro, Serra do Retiro, Estação 9, 09 $19^{\prime} 60^{\prime \prime} \mathrm{S} 38^{\circ} 18^{\prime} 35^{\prime \prime} \mathrm{W}, 545 \mathrm{~m}$, fl., 23.IV.2014, L.R. Silva et al. 283 (HUNEB); Jeremoabo, APA Serra Branca, Estrada principal em direção a Serra do Navio, 0953'20.7"S 38 40'14.9"W, 422 m, 24.I.2013, fl., L.R. Silva et al. 133 (HUNEB); Faz. Serra Branca, estrada sentido a Serra Branca, 0952'51.9"S 38 39'28.9"W, 487 m, 10.XII.2008, fl., M.V.V. Romão et al. 423 (HUNEB); Faz. Serra Branca, Trilha próximo a Serra do Navio, 0951'58.1"S 38 38'46.9"W, 482 m, fl., 03.XI.2011, fl., L.R. Silva et al. 52, 53 (HUNEB); Faz. Serra Branca, Trilha próximo a Serra do Navio, $09^{\circ} 51^{\prime} 58.1^{\prime \prime} \mathrm{S} 38^{\circ} 38^{\prime} 46.9^{\prime \prime} \mathrm{W}, 482 \mathrm{~m}$, fl., 03.XI.2011, fl., L.R. Silva et al. 52, 53 (HUNEB); Faz. Serra Branca, Trilha próximo ao tanque de dentro, próximo a Serra do Navio, 0951'56.4"S 38 38'34.2"W, 484 m, 04.XI.2010, fl., L.R. Silva et al. 01 (HUNEB).

This species is found in dry forest vegetation in Bolivia, Paraguay and Brazil (Bahia, Mato Grosso, Minas Gerais and Pernambuco) (Lohmann \& Taylor 2014). It is underrepresented in the Ecoregion, being collected only at EPA Serra Branca and Serra do Retiro in preserved Forest areas. It flowers in the month of April and also during the dry season in the months of November and December.

Bignonia convolvuloides is characterized by branches with bromeliad-like prophylls, calyx with apiculate lobes, nectariferous disk inconspicuous and papillate ovary.

3. Cuspidaria lateriflora DC., Prodr. [A. P. de Candolle]. 9: 179. 1845. Figures $2 \mathrm{C} ; 3 \mathrm{~L}-\mathrm{O}$

Lianas or scandent shrubs; branchlets cylindrical, striated, with lenticels, tomentose to glabrescent, without interpetiolar glands. Prophylls of the axillary buds minute. Leaves 3-foliolate, petioles $0.1-0.5 \mathrm{~cm}$, tomentose, petiolules ca. $0.25 \mathrm{~cm}$, pubescent, leaflets ca. 2.5 $\times 2 \mathrm{~cm}$, discolorous, chartaceous, ovate or lanceolate, apex acuminate, margins entire, base rounded, venation penninerved, adaxial surface tomentose, abaxial surface tomentose or velutinous, trichomes tector and stipitate glandular trichomes; tendrils simple, with tips not uncinate. Inflorescence a thyrse, peduncles ca. $5 \mathrm{~cm}$, tomentose, pedicel ca. 1.3 $\mathrm{cm}$, pubescent, bracts and bracteoles ca. $3 \mathrm{~mm}$. Calyx ca. $0.6 \times 0.4 \mathrm{~cm}$, green, campanulate, 5-lobed, cuspidate, without rifts, tomentose, with glandular trichomes stipitate, without nectaries; corolla $3-4 \times$ ca. 1.5 $\mathrm{cm}$, magenta with white fauces and lilac nectar guides, infundibuliform, vellutine; stamens included, dorsal filaments ca. $1.5 \mathrm{~cm}$, ventral filaments ca. $1.1 \mathrm{~cm}$, anthers ca. $8 \mathrm{~mm}, 2$-thecate, slightly pubescent, forward-curved, connective not elongated, glabrous, staminode ca. 2 $\mathrm{mm}$, glabrous; nectariferous disk conspicuous, ovary ca. $2 \times 1 \mathrm{~mm}$, 

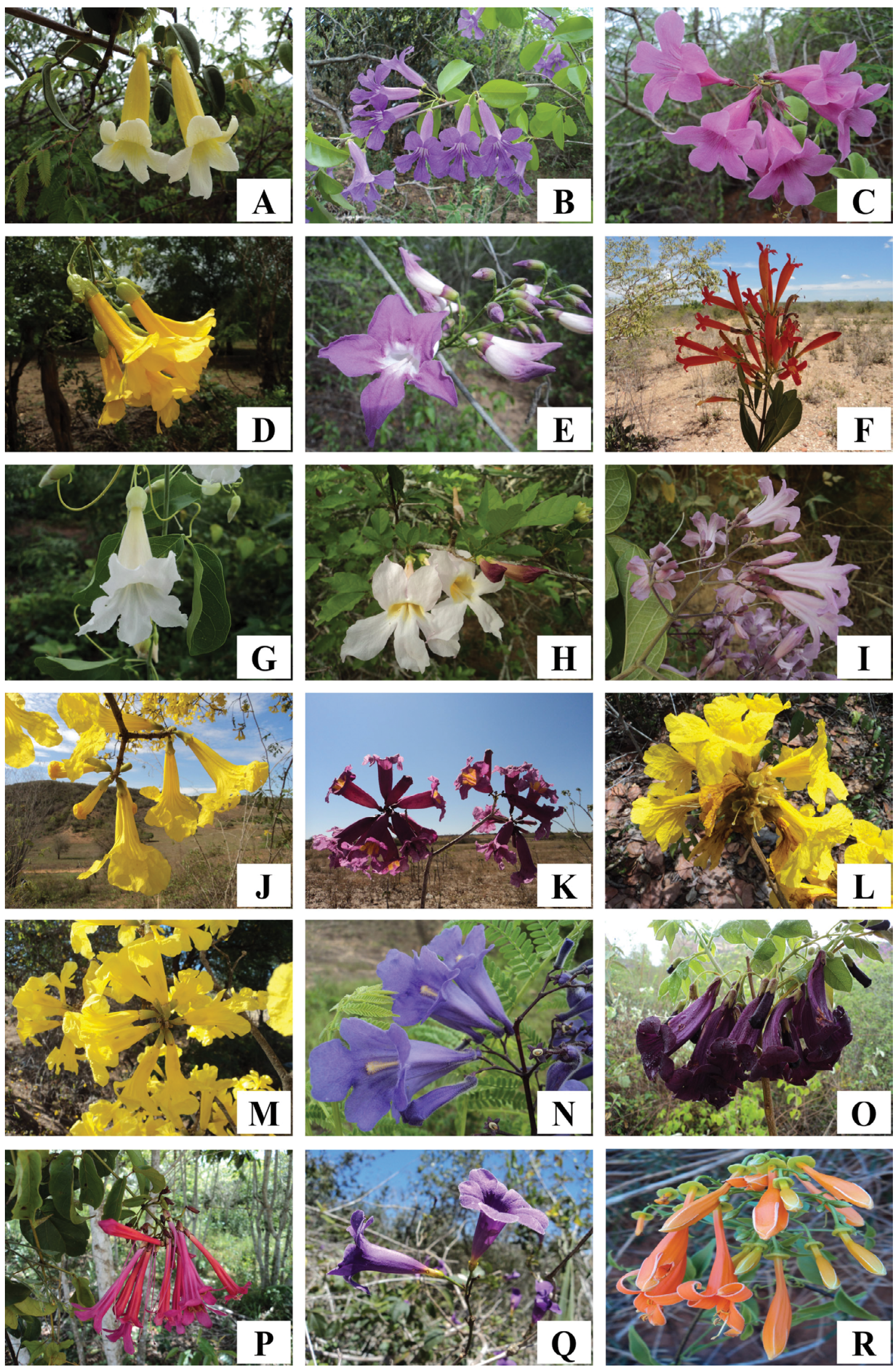

Figure 2. Representatives of the Bignoniaceae in the Raso da Catarina Ecoregion: A. Anemopaegma laeve; B. Bignonia convolvuloides; C. Cuspidaria lateriflora; D. Dolichandra quadrivalvis; E. Fridericia dichotoma; F. Fridericia erubescens; G. Fridericia limae; H. Fridericia parviflora; I. Fridericia pubescens; J. Handroanthus grandiflorus; K. Handroanthus impetiginosus; L. Handroanthus ochraceus; M. Handroanthus serratifolius; N. Jacaranda brasiliana; O. Jacaranda jasminoides; P. Lundia longa; Q. Mansoa paganuccii; R. Pyrostegia venusta. 
Bignoniaceae in the RCE
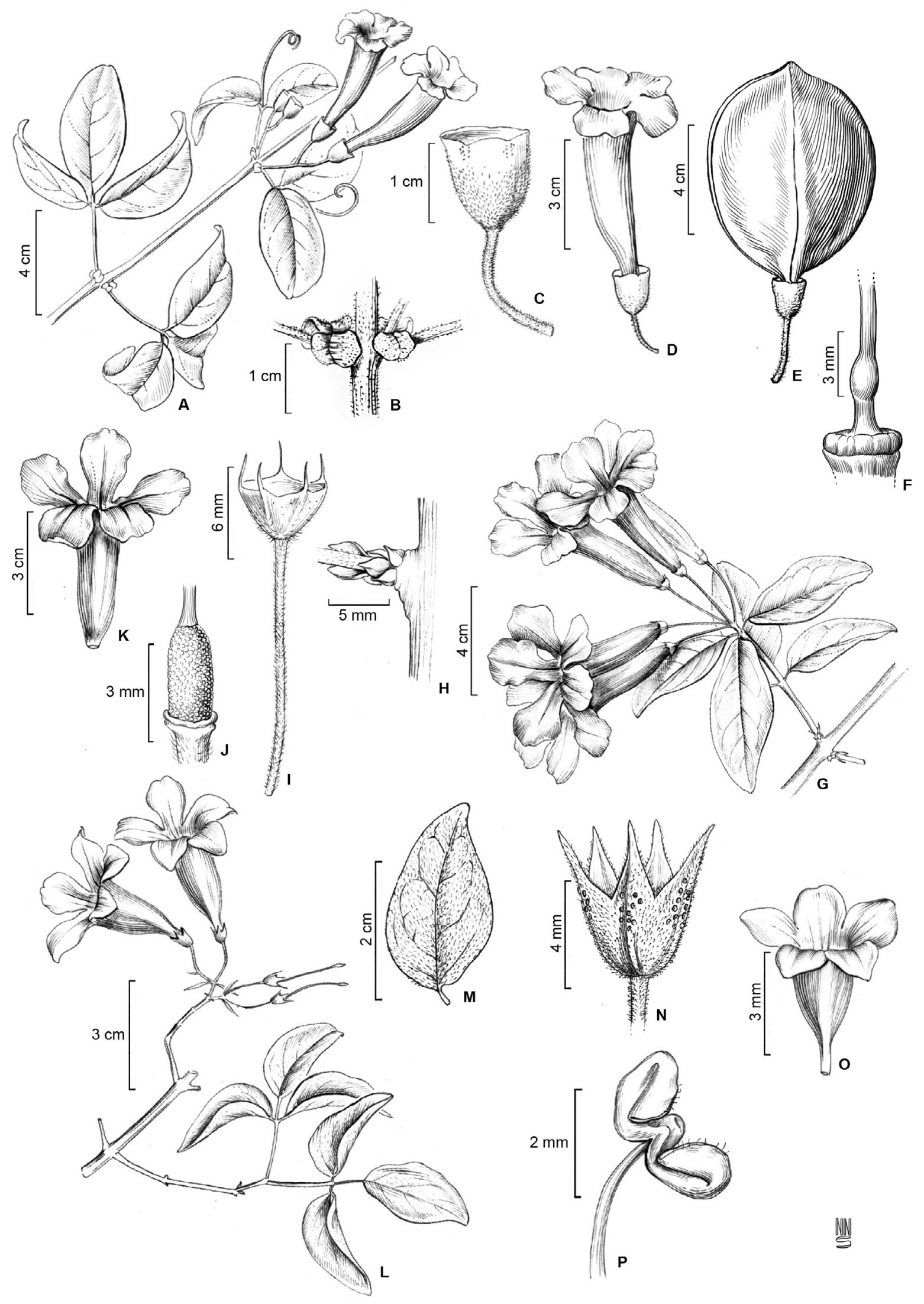

Figure 3. A-F. Anemopaegma laeve: A. flowering branch; B. prophylls of the axillary buds foliaceous; C. calyx; D. flower; E. fruit; F. ovary with nectariferous disk. G-K. Bignonia convolvuloides: G. flowering branch; H. branch detail with prophylls of the axillary buds bromeliad-like; I. calyx; J. ovary; K. corolla. L-O. Cuspidaria lateriflora: L. flowering branch; M. leaflet; N. calyx; O. corolla; P. anthers. Illustrations by Natanael Santos; A-F from R. R Varjão 19; G-K from L. R. Silva 52; L-O from L. R. Silva 08. (Silva et al. 2016). 
lepidote, sessile, not ridged, style ca. $1.4 \mathrm{~cm}$, stigma ca. $3 \mathrm{~mm}$. Capsules $30-50 \times 0.7 \mathrm{~cm}$, linear, slightly rounded, striated, coriaceous, margins flat, without wings. Seeds ca. $2.3 \times 0.7 \mathrm{~cm}$.

Material examined: BRAZIL. BAHIA: Canudos, Estação Biológica de Canudos, Base 1, 0956'59"S 3859'32"W, 397 m, 29.III. 2012, fr., D.D. Vieira et al. 240 (HUNEB); Glória, Aldeia Kataruré, próximo ao pé da serra, 0909'40"S 38²2'54"W, 635 m, 04.V.2013, fl., L.R. Silva et al. 155 (HUNEB); Jeremoabo, APA Serra Branca, Estrada que liga a Estação Ecológica Raso da Catarina ao Povoado Quelés, $09^{\circ} 53^{\prime} 01.8^{\prime \prime} \mathrm{S} 38^{\circ} 32^{\prime} 39.5^{\prime \prime} \mathrm{W}, 650 \mathrm{~m}$, 22.IX.2010, fr., D.D. Vieira et al. 80 (HUNEB); Caminho velho em direção a João Gomes, 0956'44.8"S $38^{\circ} 42^{\prime} 10.0^{\prime \prime} \mathrm{W}, 328$ m, 04.XI.2011, fl., J.B. Lima et al. 19 (HUNEB); Faz. Serra Branca, Trilha em direção ao tanque do coleta, $09^{\circ} 54^{\prime} 01.1^{\prime \prime} \mathrm{S}$ $38^{\circ} 41^{\prime} 00.7^{\prime \prime} \mathrm{W}, 399$ m, 05.X.2010, fl., L.R. Silva et al. 08 (HUNEB); Faz. Serra Branca, Trilha próximo ao curral, 0956'34.4"S 3842'01.2"W, 329 m, 27.III.2012, fr., L.R. Silva et al. 61 (HUNEB); Paulo Afonso, Estação Ecológica do Raso da Catarina, trilha antes da curva das solanáceas, 0949'05.4"S 38²9'31.2"W, 695 m, fl., L.R. Silva et al. 204 (HUNEB); Mata das Pororocas, $09^{\circ} 20^{\prime}$ S $38^{\circ} 29^{\prime} W$, 24.VI.1982, fl.; fr., L. Queiroz 294 (HRB); Raso da Catarina, 09³9'02.5”S 38²9'36.3”W, $621 \mathrm{~m}$, 09.VIII.2009, fr., M.V.V. Romão et al. 579 (HUNEB); Raso da Catarina, s/c, 14.V.1981, fr., G. Pinto 104/81 (HRB); Raso da Catarina, Cachimbo, 09³9'12"S 38 32'14"W, 595 m, 10.VIII.2005, fr., E.B. Miranda et al. 808 (HUEFS); Reserva Ecológica Raso da Catarina, s/c 6.XII.1985, fl., M.C. Guedes 987 (ALCB).

Cuspidaria lateriflora is distributed in dry to wet forest vegetation in Peru, Bolivia, Paraguay, and Brazil (Lohmann \& Taylor 2014). In Brazil, it is found from the north to the southeast, in areas of the Amazon Rainforest, Caatinga, Cerrado, and Atlantic Forest (Lohmann 2015). It can be easily found throughout the study area, registered to three conservation units: EBC, EPA Serra Branca and ESRC, with peaking bloom between November and January.

In the area, the species can be recognized by small (ca. $2.5 \times 2$ $\mathrm{cm}$ ) leaflets with tector and glandular stipitate trichomes, which are of a sticky consistency in the fields, bracts, calyx with cuspidate lobes, glandular trichomes stipitate, anthers with thecae forward-curved, slightly pubescent and capsule linear striated.

\section{Dolichandra quadrivalvis (Jacq.) L.G.Lohmann, Nuevo} Cat. Fl. Vasc. Venezuela. 273. 2008. Figures 2D; 4A-D

Lianas; branchlets cylindrical, striated, with lenticels, slightly pubescent, with interpetiolar glands. Prophylls of the axillary buds minute. Leaves 2-foliolate, petioles ca. $2 \mathrm{~cm}$, pubescent, petiolules ca. $1-1.5 \mathrm{~cm}$, slightly pubescent, leaflets $5.5-8.5 \times 3.5-6.5 \mathrm{~cm}$, concolorous, membranaceous to chartaceous, elliptic or ovate-lanceolate, apex acuminate or cuneate, margins entire, base rounded to cuneate, venation penninerved, adaxial surface glabrous with tector trichomes restricted to the midrib, abaxial surface glabrous with restricted trichomes the veins; tendrils trifid, with uncinate tips. Inflorescence a thyrse, peduncles ca. $1.5-3 \mathrm{~cm}$, pedicel ca. $1-2 \mathrm{~cm}$, both with tector trichomes sparse, bracts and bracteoles foliaceous eliptic ca. $20 \times 10 \mathrm{~mm}$. Calyx ca. 2-2.5 $\times 1$ $\mathrm{cm}$, green, spathaceous, with a recurved apicule, without rifts, glabrous, without nectaries; corolla ca. $7.5 \times 2 \mathrm{~cm}$, yellow, tubular, glabrous; stamens included, dorsal filaments ca. $2 \mathrm{~cm}$, ventral filaments ca. 1.4 $\mathrm{cm}$, anthers ca. $8 \mathrm{~mm}, 2-$ thecate, glabrous, straight, connective not elongated, glabrous, staminode ca. $8 \mathrm{~mm}$, glabrous; nectariferous disk conspicuous; ovary ca. $3 \times 2 \mathrm{~mm}$, glabrous, sessile, ridged, style ca. 3 $\mathrm{cm}$, stigma ca. $3 \mathrm{~mm}$. Fruits and seeds not seen.

Material examined: BRAZIL. BAHIA: Canudos, Estação Biológica de Canudos, $10^{\circ} 01^{\prime} 00^{\prime \prime} \mathrm{S} 39^{\circ} 09^{\prime} 00^{\prime \prime} \mathrm{W}, 25 . X I .2003$, fl., F.H.M. Silva et al. 485 (HUEFS); Glória, Brejo do Burgo, Caminho da Serrota, s/c, 03.VII.1995, fr., F.P. Bandeira 247 (HRB); Jeremoabo, Caminho sentido a Fazenda Serra Branca, em frente a casa de Dona Amelinda, 1001'29.7"S 38³2'21.7"W, 294 m, 20.XII.2013, fl., L.R. Silva et al. 248 (HUNEB); Estrada em direção ao Povoado Lagoa da Mata do Sertão, $10^{\circ} 01^{\prime 2} 29.7^{\prime \prime} \mathrm{S} 38^{\circ} 32^{\prime 2} 21.7^{\prime \prime} \mathrm{W}, 294$ m, 25.I.2013, fl., L.R. Silva et al. 137 (HUNEB); Ribeira do Pombal, Povoado do Pau Ferro, 10³9'34.6"S $38^{\circ} 40^{\prime 2} 274^{\prime \prime} \mathrm{W}, 267$ m, 15.I.2014, fl., L.R. Silva et al. 258 (HUNEB); Santa Brígida: ca. $4 \mathrm{~km} \mathrm{~W}$ do entroncamento de Santa Brígida com a BR-110, $09^{\circ} 44^{\prime}$ S $38^{\circ} 16^{\prime} \mathrm{W}$, s/d, fl., L.P. de Queiroz \& N.S. Nascimento 3741 (HUEFS).

This species is widely distributed in dry to wet forests and particularly in seasonal lowland forest vegetation, in Mexico, Guatemala, El Salvador, Honduras, Nicaragua, Costa Rica, Colombia, Venezuela, Guyana, Peru, Bolivia, Paraguay, Brazil, Uruguay and Argentina (Woodson et al. 1973, Lohmann \& Taylor 2014). In Brazil, it is widely distributed throughout the southern states to the north, in environments of Amazon Rainforest, Caatinga, Atlantic Forest and Pantanal (Lohmann 2015). In the Ecoregion was registered to two conservation units: EBC, EPA Serra Branca, in areas of open Caatinga and edges of roads. It flowers in December reaching its peak of flowering that month, extending until January.

Dolichandra quadrivalvis can be identified by tendrils trifid, with uncinate tips, bracts and bracteoles foliaceous, flowers with spathaceous calyx, with a recurved apicule and yellow corolla.

5. Fridericia dichotoma (Jacq.) L.G.Lohmann, Ann. Missouri Bot. Gard. 99: 436-437. 2014. Figures 2E; 4E-I

Lianas; branchlets cylindrical, striated, with lenticels, glabrous, without interpetiolar glands. Prophylls of the axillary buds minute. Leaves 2-3- foliolate, petioles $4-5.5 \mathrm{~cm}$, petiolules $0.2-0.8 \mathrm{~cm}$, both slightly pubescent; leaflets $5.5-7.5 \times 2.5-3.0 \mathrm{~cm}$, concolorous, membranaceous, elliptical, apex acuminatte, margins entire, base rounded or slightly cordate, venation penninerved, both surfaces with tector trichomes restricted to the midrib; tendrils simple, with tips not uncinate. Inflorescence a thyrse, peduncles ca. $1 \mathrm{~cm}$, pedicel ca. $0.5 \mathrm{~cm}$, both pubescent, bracts and bracteoles inconspicuous. Calyx ca. $0.9 \times 0.7$ $\mathrm{cm}$, green and magenta base at the apex, campanulate, truncate, without rifts, tector trichomes sparse, without nectaries; corolla 3-5 × 1-1.5 $\mathrm{cm}$, lilac, lobes magenta and fauces white, infundibuliform, tomentose; stamens included, dorsal filaments ca. $1.8 \mathrm{~cm}$, ventral filaments ca. 1.3 $\mathrm{cm}$, anthers ca. $6 \mathrm{~mm}, 2$-thecate, glabrous, straight, connective not elongated, glabrous, staminode ca. $5 \mathrm{~mm}$, glabrous; nectariferous disk conspicuous; ovary ca. $2 \times 1 \mathrm{~mm}$, lepidote, sessile, ridged, style ca. 2 $\mathrm{cm}$, stigma ca. $2 \mathrm{~mm}$. Fruits and seeds not seen.

Material examined: BRAZIL. BAHIA: Glória, Povoado Retiro, Serra do Retiro, Estação 9, fl., 23.IV.2014, L.R. Silva et al. 284 (HUNEB); Jeremoabo, APA Serra Branca, fazenda Serra Branca, caminho da vaca morta em direção a Serra Branca, 0953'43.1"S $38^{\circ} 40^{\prime} 44.6^{\prime \prime} \mathrm{W}, 430$ m, 04.XI.2010, fl., L.R. Silva et al. 06 (HUNEB); Baixa dos Quelés, 17.X.2009, fl., E. Melo et al., 6690 (ALCB); Trilha sentido a Serra do Navio, 0951'58.1"S 38 40'44.6"W, 482 m, 
Bignoniaceae in the RCE

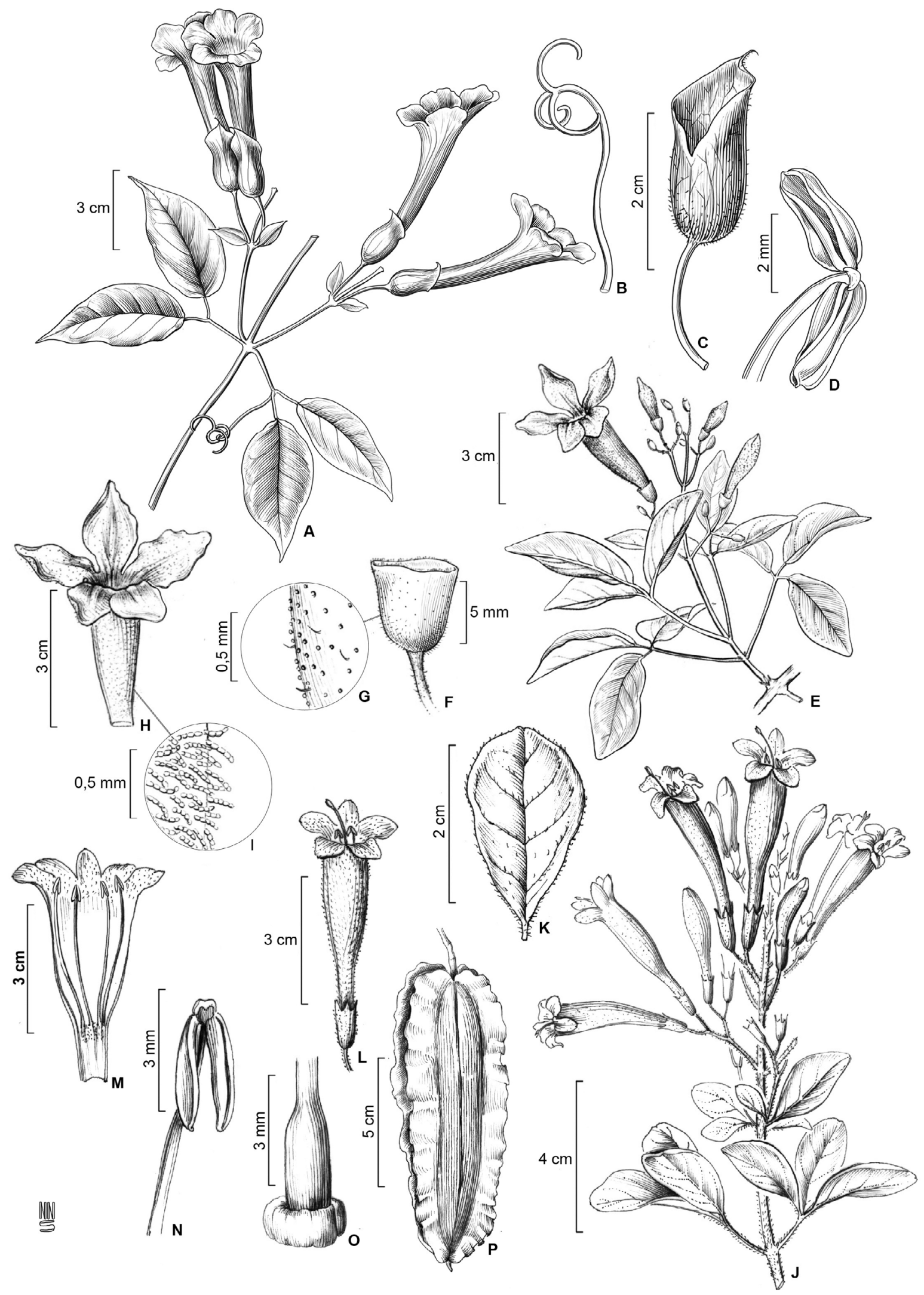

Figure 4. A-D. Dolichandra quadrivalvis: A. flowering branch; B. tendril; C. calyx; D. anthers. E-I. Fridericia dichotoma: E. flowering branch; F. calyx; G. indument of the calyx; H. corolla; I. indument of the corolla. J-P. Fridericia erubescens: J. flowering branch; K. leaflet; L. flower with stamens exserted; M. opened corolla; N. anthers; O. ovary; P. fruit. Illustrations by Natanael Santos; A-D. from L.R. Silva 137; E-I. from L.R. Silva 06; J-P. from D.D. Vieira 89. Figures E-P (Silva et al. 2016). 
03.XI.2011, fl., L.R. Silva et al. 54 (HUNEB); Paulo Afonso, Raso da Catarina, estrada para o alojamento, 09³9'12" S 38 $31^{\prime} 52^{\prime \prime} \mathrm{W}, 550 \mathrm{~m}, 10$. VIII.2005, fr., E.B. Miranda et al. 861 (HUEFS).

Fridericia dichotoma is a species widely distributed in dry to humid lowland forest, occurring from Mexico to Argentina. The species is extremely variable morphologically (Gentry et al. 1973, Lohmann \& Taylor 2014). It has records from the north to the southeast of Brazil, in environments of Amazon Rainforest, Caatinga, Cerrado, Atlantic Forest and Pantanal (Lohmann 2015). It was found in three conservation units: EBC, EPA Serra Branca and ESRC. It flowers in April and also during the dry season in the months of November and December.

Fridericia dichotoma presents membranaceous leaflets, truncate calyx, tomentose corolla, lilac with magenta lobes and white fauces.

6. Fridericia erubescens (DC.) L.G.Lohmann, Ann. Missouri Bot. Gard. 99: 437-438. 2014. Figures 2F; 4J-P

Lianas or scandent shrubs; branchlets cylindrical, without streaking, with lenticels, pilose, with interpetiolar glands. Prophylls of the axillary buds minute. Leaves 2-3-foliolate, petioles $1.5-4 \mathrm{~cm}$, petiolules $0.4-1$ $\mathrm{cm}$, both pubescent; leaflets $3-6 \times 2.5-4.5 \mathrm{~cm}$, discolorous, chartaceous, obovate, apex rounded, margins entire, base attenuate, venation penninerved, adaxial surface with sessile glandular trichomes sparse on limbo and tector trichomes concentrated on the midrib, abaxial surface pubescent; tendrils simple, with tips not uncinate. Inflorescence a thyrse, peduncles ca. $1.9 \mathrm{~cm}$, pubescent, pedicel ca. $0.5 \mathrm{~cm}$, pilose, bracts and bracteoles ca. $6 \mathrm{~mm}$. Calyx ca. $0.8 \times 0.4 \mathrm{~cm}$, green, tubular, 5-lobed, cuspidate, without rifts, slightly pubescent, with tector trichomes and glandular trichomes, without nectaries; corolla 4.5-5.5 $\times 1-1.3 \mathrm{~cm}$, red to orange passing pink after pollination, with red to orange fauces, infundibuliform, pubescent; stamens exserted, dorsal filaments ca. $4.4 \mathrm{~cm}$, ventral filaments ca. $3.5 \mathrm{~cm}$, anthers ca. $6 \mathrm{~mm}, 2$-thecate, glabrous, straight, connective not elongated, glabrous, staminode ca. $8 \mathrm{~mm}$, glabrous; nectariferous disk conspicuous, ovary ca. $3 \times 1 \mathrm{~mm}$, glabrous, sessile, not ridged, style ca. $5 \mathrm{~cm}$, stigma ca. $2 \mathrm{~mm}$. Capsules 5-11 $\times 3-4.5 \mathrm{~cm}$, oblong, flattened, smooth, coriaceous, with wavy wings. Seeds ca. $2.5 \times 1 \mathrm{~cm}$.

Material examined: BRAZIL. BAHIA: Canudos, Estação Biológica de Canudos, Barreiro próximo ao saco 2 em direção a roça de Dominguinhos, 09 $56^{\prime} 73^{\prime \prime} \mathrm{S} 39^{\circ} 00^{\prime} 79^{\prime \prime} \mathrm{W}, 417 \mathrm{~m}, 26 . \mathrm{IX} .2012$, fr., D.D. Vieira et al. 253 (HUNEB); Toca Velha, Estação Biológica de Canudos (Sede de Biodiversitas), 1001'58"S 3909'00"W, 29.X.2001, fl., L.P. de Queiroz et al. 7023 (PEUFR); 0954'S 3908'W, 13.V.2003, fl., A. Rodarte 159 (ALCB); Jeremoabo, APA Serra Branca, estrada da Estação Ecológica Raso da Catarina, 0953'01.8"S 38³2'39.5"W, 650 m, 22.IX.2010, fl.; fr., D.D. Vieira et al. 89 (HUNEB); Baixa dos Quelés, $09^{\circ} 58^{\prime} 60^{\prime \prime} \mathrm{S} 38^{\circ} 26^{\prime} 16^{\prime \prime} \mathrm{W}, 488 \mathrm{~m}, 17 . X .2009$, fl., E. Melo et al. 6653 (ALCB); Caatinga entre Jeremoabo e Cícero Dantas, s/c, 5.III.1958, fl.; fr., A. Lima 58-2893 (IPA); Caminho da Serra Branca, 0955'13.2"S $38^{\circ} 42^{\prime} 02.2^{\prime \prime} \mathrm{W}, 362$ m, fl., 08.XII.2011, L.R. Silva et al. 17 (HUNEB); Entre Cícero Dantas e Jeremoabo, s/c, 8.X.1961, fl., A. Lima 61-3944 (IPA); Estrada principal sentido a ESEC, depois da casa de Sr. Antônio Roxinho, 0957'16.1"S 38 26'23.2"W, 505 m, 19.XII.2013, fl., L.R. Silva et al. 50 (HUNEB); Faz. Serra Branca, vaca morta, 04.XI.2011, L.R. Silva et al. 58 (HUNEB); Trilha secundária a estrada principal perto da caixa d'água, 0956'36.1"S 38 27'12.1"W, 551 m, 08.IV.2011, fl., R.R. Varjão et al. 70 (HUNEB); Paulo Afonso, Estação Ecológica Raso

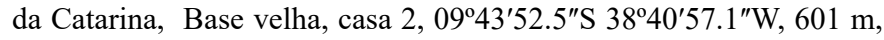
fl.; fr., L.R. Silva et al. 142 (HUNEB); Estação Ecológica do Raso da Catarina, estrada de acesso a base velha, 09 $46^{\prime} 29.0^{\prime \prime} \mathrm{S} 38^{\circ} 32^{\prime} 44.0^{\prime \prime} \mathrm{W}$, 691 m, 27.II.2013, fl., L.R. Silva et al. 140 (HUNEB); Estação Ecológica Raso da Catarina, 0940'47"S 3829'28"W, 622 m, 1.XII.2005, fl., A.O. Moraes et al. 99 (HUEFS); Estação Ecológica Raso da Catarina, beira da estrada, 0943'53"S 38 40'58"W, 541 m, 29.XI.2005, fl., R.M. Castro et al. 1275 (HUEFS); s/c, X.1949, fl., G. Pinto 49-166 (ALCB); Trilha sentido a mata da Pororoca, 09²4'39.6"S 38²9'31.1"W, 707 m, fl., L.R. Silva et al. 203 (HUNEB); Santa Brígida, ca. $50 \mathrm{~km}$ do povoado dos 40 pela estrada de terra em direção à Macambira 09 $44^{\prime} 51^{\prime \prime} \mathrm{S} 38^{\circ} 19^{\prime} 36^{\prime \prime} \mathrm{W}$, 420 m, 29.XI.2009, fl., E. Melo et al. 7334 (HUEFS); Fazenda do Sr. José Alves, 0944'15"S 38¹7'60"W, 470 m, 28.XI.2009, fl.; fr., E. Melo et al., 7291 (HUEFS).

Fridericia erubescens is endemic to Brazil, found in Cerrado, Caatinga and Atlantic Forest in the northeast and southeast regions of Brazil (Bahia, Ceará, Espírito Santo, Minas Gerais, Pernambuco, Piauí and Rio de Janeiro) (Lohmann 2015). It is a species with wide distribution in the area, registered to three conservation units: $\mathrm{EBC}$, EPA Serra Branca and ESRC. It flowers and fruiting the whole year.

In the study area is identified by obovate leaflets, the corolla is red to orange passing to pink after pollination, stamens exserted and fruits with winged margins.

\section{Fridericia limae (A. H. Gentry) L.G.Lohmann, Ann.} Missouri Bot. Gard. 99: 440. 2014. Figures 2G; 5A-F

Lianas; branchlets cylindrical, striated, with lenticels, glabrous, without interpetiolar glands. Prophylls of the axillary buds minute. Leaves 2-3-foliolate, petioles $2.5-4 \mathrm{~cm}$, petiolules ca. $1 \mathrm{~cm}$, both glabrous or slightly pubescent; leaflets $4.5-10 \times 2.5-4 \mathrm{~cm}$, concolorous, chartaceous, elliptical or oblong, apex obtuse, occasionally emarginate or retuse, margins entire, base rounded, venation penninerved, both surfaces glabrous or occasionally glabrescent, with few tector trichomes restricted to the midrib; tendrils simple, with tips not uncinate. Inflorescence a thyrse, peduncles ca. $4 \mathrm{~cm}$, pedicel ca. $2 \mathrm{~cm}$, both glabrous, bracts and bracteoles inconspicuous. Calyx ca. $1 \times 0.8 \mathrm{~cm}$, light green, slightly urceolate, truncate, without rifts, glabrous, without nectaries; corolla ca. $4 \times 1.8 \mathrm{~cm}$, white to cream with white to cream fauces, infundibuliform, pubescent; stamens included, dorsal filaments ca. $1.7 \mathrm{~cm}$, ventral filaments ca. $1.2 \mathrm{~cm}$, anthers ca. $10 \mathrm{~mm}, 2$-thecate, glabrous, straight, connective not elongated, glabrous, staminode ca. $7 \mathrm{~mm}$, glabrous; nectariferous disk conspicuous, ovary ca. $2 \times 1$ $\mathrm{mm}$, lepidote, sessile, not ridged, style ca. $2.3 \mathrm{~cm}$, stigma ca. $2 \mathrm{~mm}$. Capsules ca. $10 \times 1.5 \mathrm{~cm}$, linear, compressed, slightly rounded, smooth, coriaceous, margins constricted irregularly, without wings. Seeds ca. $3.5 \times 1.5 \mathrm{~cm}$.

Material examined: BRAZIL. BAHIA: Canudos, Reserva Biológica de Canudos, 09 58'21"S 3901'16"W, 533 m, 1.VII.2007, fr., M.M. Silva-Castro et al. 1240 (HUEFS); Glória, ca. 4 km de Olho d'Água de Souza, 09²0'37"S 38 $18^{\prime} 57^{\prime \prime} \mathrm{W}, 373$ m, 26.IV.2001, fr., L.P. de Queiroz et al. 6549 (HUEFS); Jeremoabo, APA Serra Branca, caminho que sai da Estação Ecológica em direção ao povoado Quelés, 09 $52^{\prime} 88^{\prime \prime} \mathrm{S} 38^{\circ} 32^{\prime} 70^{\prime \prime} \mathrm{W}, 653 \mathrm{~m}, 05 . \mathrm{V} .2011$, fl., D.D. Vieira et al. 148 (HUNEB); Caminho da Carnaubinha, 0957'51.5"S 38²6'11.8"W, 496 m, 19.XII.2013, fl., L.R. Silva et al. 243 (HUNEB); Depois da casa de Antônio Roxinho, 0957'16.1"S 38²6'23.2"W, 505 m, 19.XII.2013, 
Bignoniaceae in the RCE

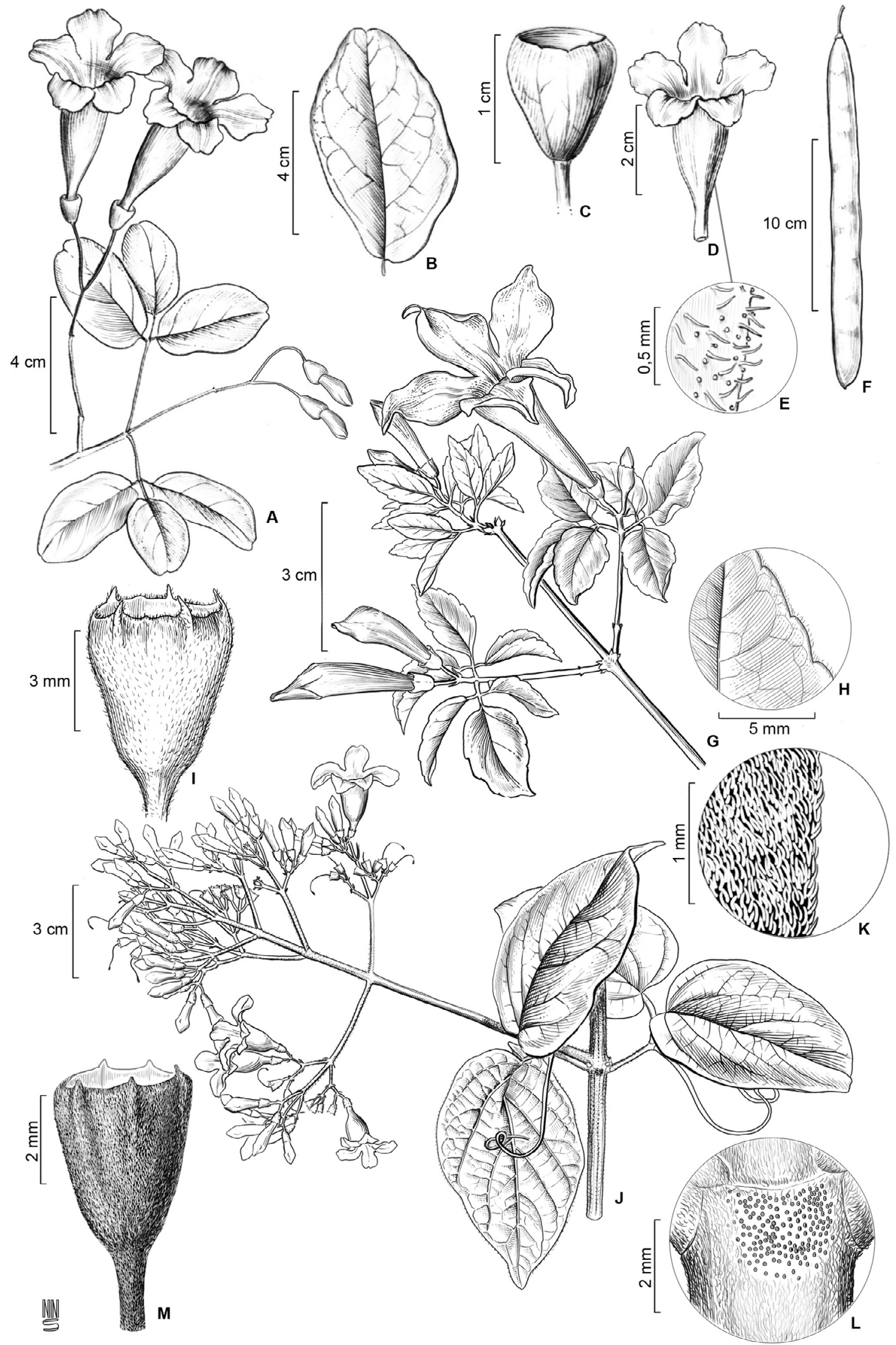

Figure 5. A-F. Fridericia limae: A. flowering branch; B. leaflet; C. calyx; D. corolla; E. indument of the corolla; F. fruit. G-I. Fridericia parviflora: G. flowering branch; H. margin of the leaflets; I. calyx. J-M. Fridericia pubescens: J. flowering branch; K. indument of the plant; L. interpetiolar glands; M. calyx. Illustrations by Natanael Santos; A-F. from L.R. Silva 31; G-I. from L.R. Silva 160; J-M. from L.R. Silva 261. Figures A-F (Silva et al. 2016). 
fl., L.R. Silva et al. 245 (HUNEB); Direita da estrada sentido a ESEC 09 $56^{\prime} 37.3^{\prime \prime S} 38^{\circ} 27^{\prime} 05.3^{\prime \prime} \mathrm{W}, 510 \mathrm{~m}, 20 . X I I .2013$, fl., L.R. Silva et al. 250 (HUNEB); Fazenda Serra Branca, caminho Serra Branca, 0954'81.3"S 38 41'65.1"W, 374 m, 08.XII.2011, fl., L.R. Silva et al. 31 (HUNEB); Paulo Afonso, Estação Ecológica Raso da Catarina 09³9'13.1"S $38^{\circ} 26^{\prime} 57.5^{\prime \prime} \mathrm{W}, 24$. XI. 2010, fl., C.L.S.B. Correia et al. 314 (HUNEB); Estação Ecológica Raso da Catarina, beira da estrada, 0943'53"S 3840'58"W, 541 m, 29.XI.2005, fl., R.M. Castro et al. 1282 (HUEFS); Estação Ecológica do Raso da Catarina, estrada da Várzea com acesso à $3^{\circ}$ casa da ESEC, $09^{\circ} 33^{\prime} 07^{\prime \prime S} 38^{\circ} 30^{\prime} 46^{\prime \prime} \mathrm{W}$, 30.I.2006, fl., A.M. Amorim et al. 5524 (HUEFS); Estação Ecológica do Raso da Catarina, estrada

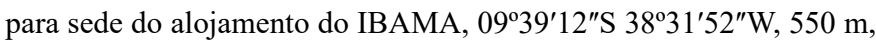
10.VIII.2005, fr., E.B. Miranda et al. 816 (HUEFS).

Fridericia limae is a species endemic from Caatinga, recorded to Bahia, Ceará, Paraíba, Pernambuco and Piauí (Lohmann 2015). In the study area can be found mainly in preserved areas, in three conservation units: EBC, EPA Serra Branca and ESRC. It reaches the peak of flowering in November and January.

The species can be recognized by chartaceous leaflets, with slightly urceolate, truncate calyx and corolla white passing to cream and linear and compressed capsule. In the field, it is also possible to smell the sweetish odor released by the flowers.

8. Fridericia parviflora (Mart. ex DC.) L.G.Lohmann, Ann. Missouri Bot. Gard. 99: 441-442. 2014. Figures 2H; 5G-I

Scandent shrubs; branchlets cylindrical, with lenticels, glabrescent, striated, without interpetiolar glands. Prophylls of the axillary buds minute. Leaves $2-3$-foliolate, petioles $0.5-1 \mathrm{~cm}$, petiolules $0.2-0.5 \mathrm{~cm}$, both pubescent; leaflets $3.5-4.0 \times 1-1.5 \mathrm{~cm}$, concolorous, membranaceous, elliptical or oblong, apex acuminate or cuneate, margins crenate, base cuneate or rounded, venation penninerved, adaxial surface slightly pubescent with tector trichomes concentrated on the midrib, abaxial surface pubescent; tendrils simple, with tips not uncinate. Inflorescence a thyrse, peduncles ca. $0.5 \mathrm{~cm}$, pedicel ca. $0.5 \mathrm{~cm}$, both pubescent, bracts and bracteoles ca. $3 \mathrm{~mm}$. Calyx ca. $0.4 \times 0.4 \mathrm{~cm}$, light green, campanulate to slightly urceolate, 5-lobed, apiculate, without rifts, pubescent, without nectaries; corolla ca. $5 \times 4 \mathrm{~cm}$, white passing the lilac, infundibuliform, pubescent; stamens included, dorsal filaments ca. $1.5 \mathrm{~cm}$, ventral filaments ca. $1 \mathrm{~cm}$, anthers ca. $10 \mathrm{~mm}, 2$-thecate, glabrous, straight, connective not elongated, glabrous, staminode ca. $4 \mathrm{~mm}$, glabrous; nectariferous disk conspicuous, ovary ca. $2 \times 1 \mathrm{~mm}$, pubescent, sessile, not ridged, style ca. $2.5 \mathrm{~cm}$, stigma ca. $2 \mathrm{~mm}$. Fruits and seeds not seen.

Material examined: BRAZIL. Bahia: Canudos, Estação Biológica de Canudos, 09 $01^{\prime} 00^{\prime \prime} \mathrm{S} 39^{\circ} 09^{\prime} 00^{\prime \prime} \mathrm{W}, 22 . X I .2003$, fl, F.H.M. Silva et al. 476 (HUEFS); Glória, Povoado Retiro, Serra do Retiro, Estação 9, $09^{\circ} 19^{\prime} 60^{\prime \prime} \mathrm{S} 38^{\circ} 18^{\prime} 35^{\prime \prime} \mathrm{W}, 545$ m, fl., 23.IV.2014, L.R. Silva et al. 282 (HUNEB); Jeremoabo, Povoado Lagoa do Sertão rumo a Jeremoabo, $10^{\circ} 01^{\prime} 51.1^{\prime \prime S} 38^{\circ} 30^{\prime} 18.1^{\prime \prime} \mathrm{W}, 319$ m, fl., 10.V.2013, L.R. Silva et al. 165-166 (HUNEB); Tucano, Sítio do Mandacaru. Faz. de Sr. Gildásio, $10^{\circ} 52^{\prime 2} 9^{\prime \prime} \mathrm{S} 38^{\circ} 46^{\prime} 19^{\prime \prime} \mathrm{W}, 391-411$ m, 15.IV.2006, fr., D. Cardoso et al. 1184 (HUEFS).

This species is endemic from Brazil, recorded to Alagoas, Bahia, Ceará, Paraíba, Pernambuco, Rio Grande do Norte, Sergipe, Mato Grosso do Sul and Minas Gerais (Lohmann \& Taylor 2014, Lohmann
2015). It is distributed through environments of Caatinga, Cerrado and Atlantic Forest (Lohmann 2015). The species is underrepresented in the study area, it was collected three times and recorded only to one conservation units: EBC. It flowers from April to May.

Fridericia parviflora is easily recognized by leaflets with membranaceous consistency and mainly by crenate margins.

\section{Fridericia pubescens (L.) L.G.Lohmann, Ann. Missouri} Bot. Gard. 99: 443-444. 2014. Figures 2I; 5J-M

Lianas; branchlets cylindrical, striated, with lenticels, densely pubescent, with interpetiolar glands conspicuous. Prophylls of the axillary buds minute. Leaves $2-$ foliolate, petioles $1-2.5 \mathrm{~cm}$, petiolules ca. $1 \mathrm{~cm}$, both pubescent; leaflets $6-7.5 \times 3-4.5 \mathrm{~cm}$, strongly discolorous, chartaceous to membranaceous, ovate or rarely elliptical, apex acuminatte, margins entire, base rounded or slightly cordate, venation penninerved, both surfaces pubescent, more intensely on the abaxial, with tector trichomes; tendrils simple with tips not uncinate. Inflorescence a thyrse, peduncles $2.5-3.5 \mathrm{~cm}$, pedicel ca. $0.2 \mathrm{~cm}$, both pubescent, bracts and bracteoles ca. $2 \mathrm{~mm}$. Calyx ca. $0.5 \times 0.3$ $\mathrm{cm}$, magenta to vinaceous, cupular, 5-lobed, apiculate, without rifts, pubescent, with glandular trichomes, without nectaries; corolla 2-2.5 $\times 1-1.5 \mathrm{~cm}$, lilac with fauces white, infundibuliform, pubescent, with glandular trichomes; stamens included, dorsal filaments ca. $1.5 \mathrm{~cm}$, ventral filaments ca. $1 \mathrm{~cm}$, anthers ca. $5 \mathrm{~mm}, 2$-thecate, glabrous, straight, connective not elongated, glabrous, staminode ca. $6 \mathrm{~mm}$, glabrous; nectariferous disk conspicuous, ovary ca. $2 \times 1 \mathrm{~mm}$, lepidote, sessile, not ridged, style ca. $1.1 \mathrm{~cm}$, stigma ca. $1 \mathrm{~mm}$. Fruits and seeds not seen.

Material examined: BRAZIL. BAHIA: Ribeira do Pombal, Banzaê, Povoado do Salgado, estrada do pau-branco, 10³5'55.1"S 38 41'55.8"W, 306 m, 15.I.2014, fl., L.R. Silva et al. 261 (HUNEB).

Fridericia pubescens spreads in dry to humid forest vegetation distributed from Mexico to Brazil (Lohmann \& Taylor 2014), found in Amazon Rainforest, Caatinga, Cerrado, Atlantic Forest and Pantanal (Lohmann 2015). It was collected only once in the municipality of Ribeira do Pombal in an open field. It was found with flowers in January.

In the study area it can be differentiated from the other congeners because it presents pubescence distributed throughout the plant, interpetiolar glands conspicuous, leaflets strongly discolorous with abaxial face densely pubescent and small corolla of $2-2.5 \times 1-1.5 \mathrm{~cm}$.

\section{Handroanthus chrysotrichus (Mart. ex DC.) Mattos,} Loefgrenia. 50: 4. 1970. Iconography: Espírito Santo (2013: 13(10).

Tree ca. $15 \mathrm{~m}$; branchlets cylindrical to sub-tetragonal, slightly streaking, without lenticels, tomentose when young, without interpetiolar glands. Prophylls of the axillary buds minute. Leaves digitate, (3-)5-foliolate, petioles 1.9-6.3(-10.4) $\mathrm{cm}$, petiolules $0.6-2.3(-3.4) \mathrm{cm}$, both stellate-tomentose; leaflets $4-8.5(-15.3) \times$ 2.9-4.4(-7.9) cm, discolorous, membranaceous to chartaceous, obovate, apex obtuse to acuminate, margins entire, rarely irregularly serrated in the medial apical portion, base cuneate to truncate, occasionally cordate, venation penninerved, adaxial surface green, sparsely lepidote and stellate-tomentose, abaxial surfaces cream-ferruginous, densely stellate-tomentose, with tector trichomes. Inflorescence a panicle, 
peduncles inconspicuous, flowers subsessile or with the pedicel to $0.5 \mathrm{~cm}$, densely tomentose, bracts and bracteoles 2-6 mm. Calyx ca. $1.5 \times 0.8 \mathrm{~cm}$, green-ferruginous, campanulate, 5-lobed, acute lobes, without rifts, densely vilose, trichomes stellate and dendroid, without nectaries; corolla $6-7 \times$ ca. $3 \mathrm{~cm}$, yellow, infundibuliform, glabrous; stamens included, dorsal filaments ca. $1.8 \mathrm{~cm}$, ventral filaments ca. 1.3 $\mathrm{cm}$, anthers ca. $5 \mathrm{~mm}, 2-$ thecate, glabrous, straight, connective not elongated, glabrous, staminode ca. $5 \mathrm{~mm}$, glabrous; nectariferous disk conspicuous, ovary ca. $5 \times 2 \mathrm{~mm}$, pilose, sessile, without ridged, style ca. $2.5 \mathrm{~cm}$, stigma ca. $2 \mathrm{~mm}$. Fruits and seeds not seen.

Material examined: BRAZIL. BAHIA: Jeremoabo, APA Serra Branca, Estrada para os Quelés, 09 $57^{\prime} 46.4^{\prime \prime} \mathrm{S} 38^{\circ} 25^{\prime} 36.2^{\prime \prime} \mathrm{W}, 523 \mathrm{~m}$, 23.I.2013, fl., L.R. Silva et al. 127 (HUNEB).

The species occurs in the northeast, southeast and south of Brazil with records Cerrado and Atlantic Forest (Lohmann 2015), being distributed by the coastal region of Brazil, recorded for restingas, open forests and disturbed forests, widely used as ornamental (Gentry, 1992b). In the Ecoregion the species is underrepresented, collected only once with flower and few very young leaves, in an area of slope. It was collected with flowers only in January.

In the study area, the species can be confused with Handroanthus ochraceus, mainly because both present rust green goblet. However, $H$. chrysotrichus can be differentiated by presenting leaflets with a rusty abaxial face (vs. leaflets with abaxial cream whitish in H. ochraceus). According to Gentry (1992), H. chrysotrichus as Tabebuia chrysotricha, could be treated as a subspecies of $H$. ochraceus. The occurrence of intermediate forms between the two species may make it difficult to distinguish the taxa (Espírito Santo et al. 2013).

\section{Handroanthus grandiflorus F.Esp.Santo \& M.M.Silva,} Phytotaxa. 48: 2. 2012. Figures 2J; 6A-F

Tree ca. $12 \mathrm{~m}$; branchlets cylindrical, without streaking, with lenticels, glabrescent, without interpetiolar glands. Prophylls of the axillary buds minute. Leaves digitate, 3-4-foliolate, petioles $4-6 \mathrm{~cm}$, petiolules $2.5-3 \mathrm{~cm}$, both slightly pubescent, with tector trichomes and glandular sessile; leaflets 9-16.5 × 4-7 cm, concolorous, chartaceous, elliptical, apex acute to acuminate, margin entire or crenulate at the middle apical portion, base cuneate to rounded, venation penninerved, adaxial surface glabrescent, abaxial surface glabrescent with tector and stellate trichomes restricted on the midrib. Inflorescence a raceme, peduncles inconspicuous, pedicel ca. $0.5 \mathrm{~cm}$, densely tomentose, bracts and bracteoles ca. $2 \mathrm{~mm}$. Calyx ca. $1.7 \times 1.5 \mathrm{~cm}$, green passing the yellow, broadly campanulate, 3-4-lobed, irregularly divided, without rifts, pubescent at the base passing sparse towards the apex, trichomes stellate, also sparse-lepidote, without nectaries; corolla ca. $11 \times 3 \mathrm{~cm}$, yellow, infundibuliform, glabrous; stamens included, dorsal filaments ca. $2.5 \mathrm{~cm}$, ventral filaments ca. $1.5 \mathrm{~cm}$, anthers ca. $6 \mathrm{~mm}, 2$-thecate, glabrous, straight, connective not elongated, glabrous, staminode ca. $5 \mathrm{~mm}$, glabrous; nectariferous disk conspicuous, ovary ca. $7 \times 2 \mathrm{~mm}$, glandular, sessile, ridged, style ca. $2.8 \mathrm{~cm}$, stigma ca. $3 \mathrm{~mm}$. Fruits and seeds not seen.

Material examined: BRAZIL. BAHIA: Jeremoabo, Caminho a esquerda do Enterolobium, 0957'72.9"S 38²5'87.1"W, 499 m, 23.X.2013, fl., L.R. Silva et al. 231 (HUNEB); Estrada em direção ao Povoado Quelés, próximo a casa de Sr. Antônio Roxinho, 0957'92.7"S $38^{\circ} 26^{\prime 2} 21.3^{\prime \prime} \mathrm{W}, 485$ m, 23.X.2013, fl., L.R. Silva et al. 232 (HUNEB).
Handroanthus grandiflorus is a species critically endangered, endemic from Caatinga, with records for the state of Bahia, its geographic distribution can also be used to distinguish it from the morphologically most similar species, which predominantly occur in more humid regions (Espírito Santo et al. 2012). In the study area it is underrepresented, with only two individuals registered. It was collected with flowers in October.

In the Ecoregion the species can be confused with other congeners that also present yellow flowers. However, it differs from the flowers with a broadly campanulate calyx, sparsely stellate-tomentose and corolla with ca. $11 \mathrm{~cm}$.

\section{Handroanthus impetiginosus (Mart. ex DC.) Mattos,} Loefgrenia. 50: 2. 1970. Figures 2K; 6G-J

Tree ca. $9 \mathrm{~m}$; branchlets cylindrical, without streaking, without lenticels, glabrous, without interpetiolar glands. Prophylls of the axillary buds minute. Leaves digitate, 5-foliolate, petioles $5-8.5 \mathrm{~cm}$, petiolules $1-4 \mathrm{~cm}$, both glabrous; leaflets $7-10 \times 2.5-4 \mathrm{~cm}$, concolorous, after dried becomes blackened, chartaceous, lanceolate, apex acute, occasionally with slightly serrated apex, margins entire, base rounded or cuneate, venation penninerved, adaxial surface glabrescent, abaxial surfaces with tector trichomes and dendroid. Inflorescence a thyrse, peduncles ca. 1 $\mathrm{cm}$, pedicel ca. $1 \mathrm{~cm}$, both glabrous, bracts and bracteoles inconspicuous. Calyx ca. $0.6 \times 0.5 \mathrm{~cm}$, green to magenta, tubular to campanulate, 5-lobed, irregular lobes, without rifts, glabrous, with nectaries; corolla ca. $6.5 \times 1.5$ $\mathrm{cm}$, magenta with yellow nectar guides on the fauces, infundibuliform, slightly pubescent; stamens included, dorsal filaments ca. $2.2 \mathrm{~cm}$, ventral filaments ca. $1.3 \mathrm{~cm}$, anthers ca. $6 \mathrm{~mm}, 2$-thecate, glabrous, straight, connective not elongated, glabrous, staminode ca. $10 \mathrm{~mm}$, glabrous; nectariferous disk conspicuous, ovary ca. $3 \times 1 \mathrm{~mm}$, lepidote, sessile, ridged, style ca. $2.7 \mathrm{~cm}$, stigma ca. $3 \mathrm{~mm}$. Capsules 20-26 × 0.8-1 cm, linear, rounded, smooth, coriaceous, margins flat, without wings.

Material examined: BRAZIL. Bahia: Glória, Serra do Salgado, 09008'32"S 38 20'11"W, 337 m, 07.IX.2007, fr., A.S. Conceição et al. 1144 (HUEFS); Jeremoabo, APA Serra Branca, Estrada seguindo para Itapicuru d'água ao lado de uma casa abandonada, 09²7'19.5"S $38^{\circ} 18^{\prime} 16.8^{\prime \prime} \mathrm{W}, 388 \mathrm{~m}, 19 . X I I .2012$, fl.; fr., L.R. Silva et al. 100 (HUNEB); Estrada para os Quelés, 09 $57^{\prime} 47.3^{\prime \prime S} 38^{\circ} 25^{\prime} 36.2^{\prime \prime} \mathrm{W}, 523$ m, 23.I.2013, fl., L.R. Silva et al. 126 (HUNEB); Faz. Serra Branca, Caminho da vaca morta em direção a Serra Branca, 0953'43.1"S $38^{\circ} 40^{\prime} 44,6^{\prime \prime} \mathrm{W}, 430$ m, 04.XI.2010, fl., L.R. Silva et al. 05 (HUNEB); Faz. Serra Branca, Vaca morta, 0954'48.1"S 38 41'37.9"W, 382 m, 01.XI.2008, fl., M.V.V. Romão et al. 415 (HUNEB); Povoado Arrasta Pé, beira da estrada, $09^{\circ} 32^{\prime} 01.3^{\prime \prime} \mathrm{S} 38^{\circ} 18^{\prime} 24^{\prime \prime} \mathrm{W}, 574$ m, 25.IX.2013, fl., L.R. Silva et al. 205 (HUNEB); 10¹1'06"S 38 34'58"W, 424 m, 9.XII.2013, fl., H.A. Ogasawara \& P. Oliveira 384 (ALCB); Paulo Afonso, km 41 Canindé-Paulo Afonso (SE), 0960'83"S 3709'00"W, 19.I.1985, fl., G. Fotius 3918 (HUEFS).

The species is registered from northwestern Mexico to northwestern Argentina, mainly in deciduous and seasonly dry forests (Gentry 1992b). In Brazil, it is widely distributed from the north to the southeast, in environments of Amazon Rainforest, Caatinga, Cerrado, Atlantic Forest and Pantanal (Lohmann 2015). In the study area is commonly found, and is also widely used as ornamental. Flowers and fruit in October and January reaching peak of flowering in the months of November and December. 


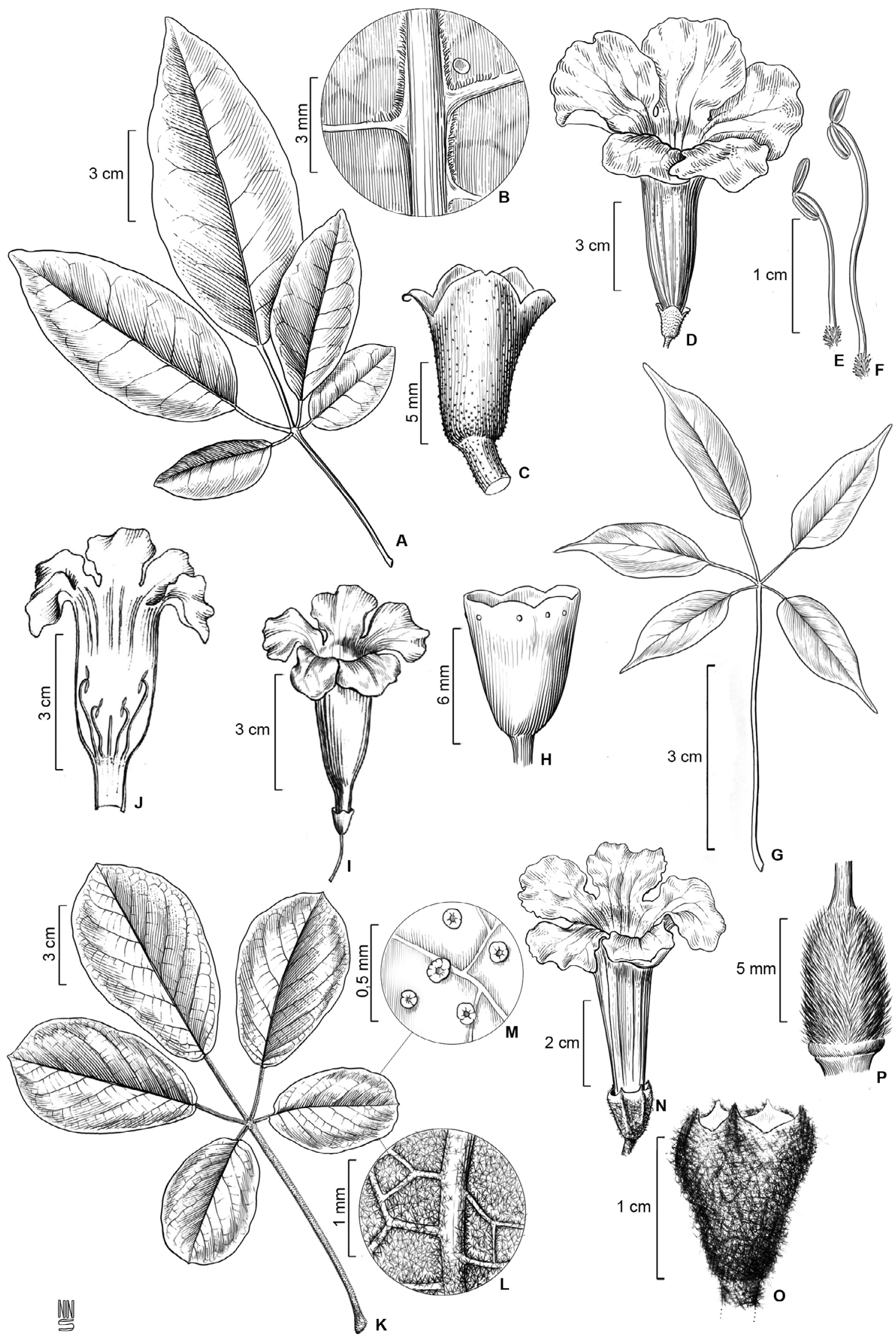

Figure 6. A-F. Handroanthus grandiflorus: A. leaf; B. abaxial surface; C. calyx; D. flower; E. ventral stamens; F. dorsal stamens. G-J. Handroanthus impetiginosus: G. leaf; H. calyx; I. flower. J. opened corolla. K-P. Handroanthus ochraceus: K. leaf; L. indument of the abaxial surface; M. indument of the adaxial surface; N. flower; O. calyx; P. ovary. Illustrations by Natanael Santos; A-F. from L.R. Silva 231; G-J from. L.R. Silva 05; K-P. from L.R. Silva 102-170. Figures G-J (Silva et al. 2016). 
Handroanthus impetiginosus is recognized by the calyx glabrous with nectaries, and magenta corolla with yellow nectar guides. This species presents morphological variation, as well as blackened leaves after drying (Espírito Santo et al. 2013).

\section{Handroanthus ochraceus (Cham.) Mattos, Loefgrenia.} 50: 2. 1970. Figures 2L; 6K-P

Tree ca. $6 \mathrm{~m}$.; branchlets cylindrical to sub-tetragonal, streaking, without lenticels, glabrescent when adult, without interpetiolar glands. Prophylls of the axillary buds minute. Leaves digitate, 5- foliolate, petioles $6-10 \mathrm{~cm}$, petiolules $1-3 \mathrm{~cm}$ both stellate-tomentose; leaflets 6-9 $\times 3.5-4.5 \mathrm{~cm}$, discolorous, chartaceous, obovate to elliptical, apex obtuse to cuspidate, margins entire passing to sinuous at the middle to the apical portion, base truncate to rounded, occasionally cordate, venation penninerved, adaxial surface green, lepidote and occasionally stellate-tomentose, abaxial surfaces whitish-cream, densely stellatetomentose, with tector trichomes. Inflorescence a panicle, peduncles inconspicuous or ca. $1 \mathrm{~cm}$, pedicel ca. $1 \mathrm{~cm}$, densely tomentose, bracts and bracteoles $4-7 \mathrm{~mm}$. Calyx ca. $1.6 \times 1 \mathrm{~cm}$, green-ferruginous, campanulate, 5-lobed, acute lobes, without rifts, densely vilose, trichomes stellate and dendroid, without nectaries; corolla ca. $7 \times 2.5$ $\mathrm{cm}$, yellow with redish nectar guides on the fauces, infundibuliform, glabrous or lepidote; stamens included, dorsal filaments ca. $2 \mathrm{~cm}$, ventral filaments ca. $1.3 \mathrm{~cm}$, anthers ca. $6 \mathrm{~mm}, 2$-thecate, glabrous, straight, connective not elongated, glabrous, staminode ca. $4 \mathrm{~mm}$, glabrous; nectariferous disk conspicuous, ovary ca. $4 \times 1.3 \mathrm{~mm}$, pilose, sessile, without ridged, style ca. $2.3 \mathrm{~cm}$, stigma ca. $2 \mathrm{~mm}$. Fruits and seeds not seen.

Material examined: BRAZIL. Bahia: Euclides da Cunha, 10³0' S $30^{\circ} 00^{\prime} \mathrm{W}$, 2004, fr., M.L. Guedes 11190 (ALCB); Jeremoabo, Baixa da forra $10^{\circ} 00^{\prime} 04.6^{\prime \prime S} 38^{\circ} 28^{\prime} 46.4^{\prime \prime} \mathrm{W}, 562$ m, 23.X.2013, fl., L.R. Silva et al. 102-170 (HUNEB).

The species is widely distributed from El Salvador to Northwest Argentina (Gentry 1992b). In Brazil It occurs from the north to the south of Brazil, in environments of Amazon Rainforest, Atlantic Forest, Caatinga and Cerrado (Lohmann 2015). In the studied ecoregion, it is poorly represented, having been collected only twice. It was collected with flowers in October.

In the study area, $H$. ochraceus is recognized by obovate to elliptic discolorous leaflets, with adaxial surface green and lepidote, abaxial surfaces whitish-cream, densely stellate-tomentose and greenferruginous calyx, densely vilose, with trichomes stellate and dendroid.

14. Handroanthus serratifolius (Vahl) S.O.Grose, Syst. Bot. 32: 666. 2007. Figures 2M; 7A-E

Tree ca. $12 \mathrm{~m}$; branchlets cylindrical, streaking, with lenticels, glabrous, without interpetiolar glands. Prophylls of the axillary buds minute. Leaves digitate, 3-5-foliolate, petioles 3-6 cm, petiolules 1-3 $\mathrm{cm}$, both pubescent, leaflets $2.5-5.5 \times 8-13.5 \mathrm{~cm}$, slightly discolorous, chartaceous, elliptical to elliptical-obovate, apex acuminate, margins entire passing to the serrated at the apex, base cuneate, venation penninerved, adaxial surface lepidote, abaxial surfaces lepidote with trichomes restricted tectores the midrib or between the midrib and secondary. Inflorescence a panicle, peduncles inconspicuous, pedicel ca. $0.5 \mathrm{~cm}$, pubescent, bracts and bracteoles ca. $2 \mathrm{~mm}$. Calyx $0.7-1$ $\times 0.5 \mathrm{~cm}$, green passing the yellow, campanulate, $3-5$ lobed, cuneate, without rifts, glabrous to sparse pubescent, tector trichomes and adpressed stellate, without nectaries; corolla $6-8 \times$ ca. $1.5 \mathrm{~cm}$, yellow, tubular-infundibuliform, glabrescent; stamens included, dorsal filaments ca. $2.1 \mathrm{~cm}$, ventral filaments ca. $1.5 \mathrm{~cm}$, anthers ca. $6 \mathrm{~mm}, 2$-thecate, glabrous, straight, connective not elongated, glabrous, staminode ca. $6 \mathrm{~mm}$, glabrous; nectariferous disk conspicuous, ovary ca. $5 \times 2 \mathrm{~mm}$, glandular-verrucose, sessile, ridged, style ca. $2.7 \mathrm{~cm}$, stigma ca. $3 \mathrm{~mm}$. Fruits and seeds not seen.

Material examined: BRAZIL. BAHIA: Itapicuru d'água, estrada entre Jeremoabo e Paulo Afonso, em frente ao restaurante da Lúcia ao lado da BR, 09 $58^{\prime} 42,2^{\prime \prime} \mathrm{S} 38^{\circ} 18^{\prime} 64,1^{\prime \prime} \mathrm{W}, 319$ m, 19.XII.2012, fl., L.R. Silva et al. 99 (HUNEB).

The species is found from Colombia to Bolivia, Guianas and Brazil. In Brazil, it has a wide distribution from the north to the south, with records for the Amazon Rainforest, Caatinga, Cerrado, Atlantic Forest and Pantanal (Gentry 1992b, Lohmann 2015). It is underrepresented in the study area, with only one individual registered. It was collected with flowers in December.

Handroanthus serratifolius can be easily identified by lepidote leaflets with trichomes restricted tectores the midrib or between the midrib and secondary, margins entire passing to the serrated at the apex, glabrous calyx to sparse pubescent, tector trichomes and adpressed stellate and ovary glandular-verrucose. According to Espírito Santo et al. (2013) it is a polymorphic species.

\section{Jacaranda brasiliana (Lam.) Pers., Syn. P1. [Persoon]. 2:} 174. 1806. Figures 2N; 7F-J

Tree ca. $10 \mathrm{~m}$; branchlets cylindrical, streaking, with lenticels, glabrescent, or with tector trichomes, without interpetiolar glands. Prophylls of the axillary buds minute. Leaves bipinnate 8-14- pairs of pinnae; rachis winged, pubescent; petioles $3-5 \mathrm{~cm}$, with tector trichomes, leaflets $0.6-1 \times 0.2-0.4 \mathrm{~cm}$, sessile, $33-45$ by pinna, strongly discolorous, chartaceous, elliptical to oblong, occasionally obovate, apex acute or acuminate, margins entire, base asymmetric cuneate, venation penninerved, adaxial surface inconspicuous, scabro with tector trichomes, abaxial surface villose, with glandular trichomes. Inflorescence a thyrse, peduncles ca. $1.8 \mathrm{~cm}$, pedicel ca. $0.6 \mathrm{~cm}$, both pubescent with glandular trichomes, bracts and bracteoles not seen. Calyx ca. $0.5 \times 0.2 \mathrm{~cm}$, violet, deeply lobed, 5-lobed, ovate, without rifts, with glandular trichomes, without nectaries; corolla 5-5.5 $\times$ ca. $3 \mathrm{~cm}$, purple to blue with white nectar guides on the fauces, tubularcampanulate, with glandular trichomes sparse; stamens included, dorsal filaments ca. $1.6 \mathrm{~cm}$, ventral filaments ca. $1 \mathrm{~cm}$, anthers ca. 3 $\mathrm{mm}, 1$-thecate, glabrous, straight, connective not elongated, glabrous, staminode ca. $28 \mathrm{~mm}$, glandular-pubescent; nectariferous disk conspicuous, ovary ca. $1 \times 2 \mathrm{~mm}$, glabrous, sessile, without ridged, style ca. $1.8 \mathrm{~cm}$, stigma ca. $2 \mathrm{~mm}$. Capsules 8-12 $\times 7-9 \mathrm{~cm}$, oblongorbicular, compressed, corrugated, woody, margins broadly undulating, without wings. Seeds ca. $3 \times 1.5 \mathrm{~cm}$.

Material examined: BRAZIL. BAHIA: Ribeira do Pombal, Banzaê, Fazenda Pedra Furada, 10³3'28.9"S 38³8'09.4"W, 333 m, 15.I.2014, fl.; fr., L.R. Silva et al. 262 (HUNEB); Fazenda Pedra Furada, 10³3'28.9"S 38 38'09.4"W, 333 m, 15.I.2014, fl., J.B. Lima et al. 161 (HUNEB). 


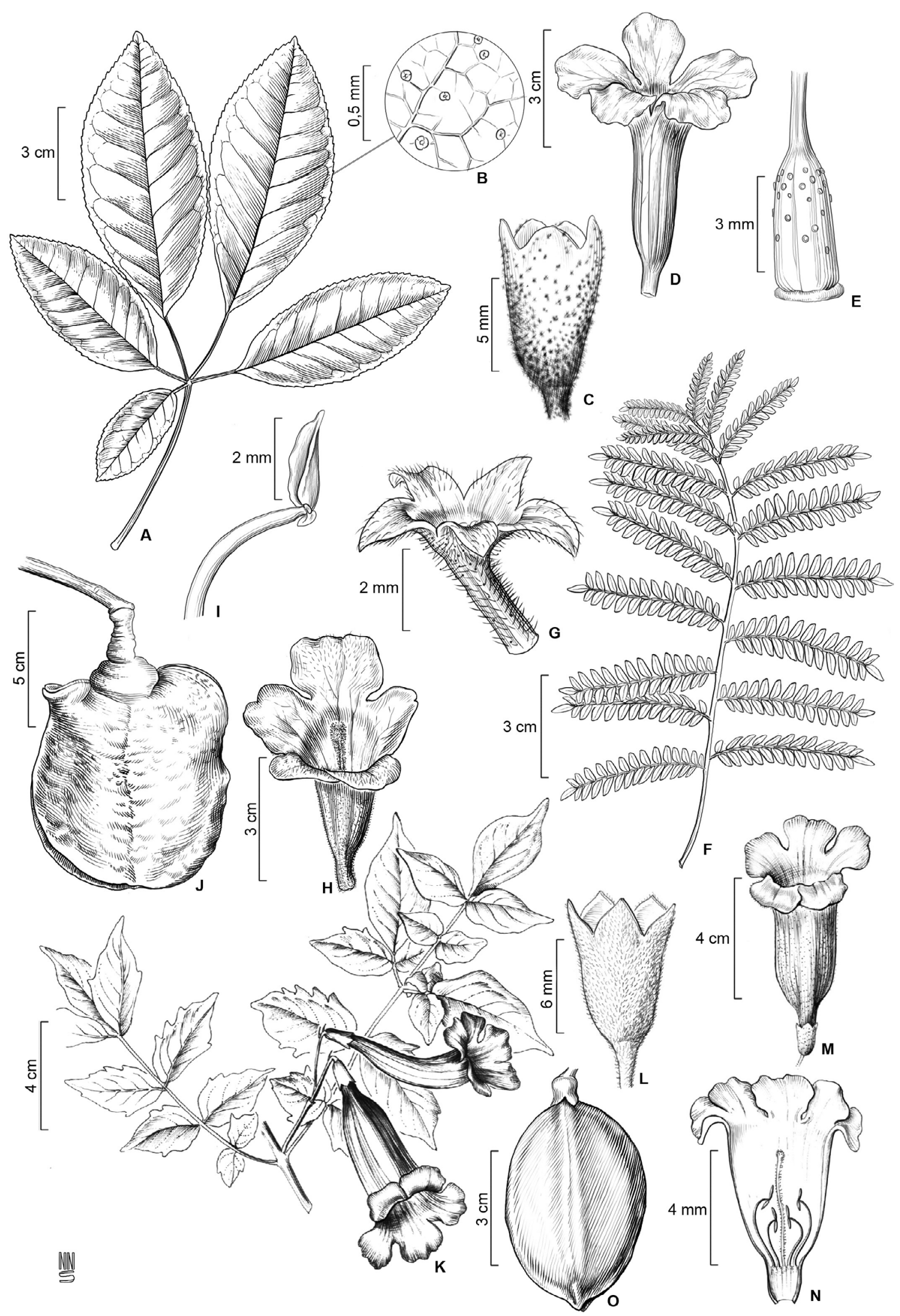

Figure 7. A-E. Handroanthus serratifolius: A. leaf; B. indument of the both surface; C. calyx; D. corolla; E. ovary. F-J. Jacaranda brasiliana: F. leaf; G. calyx; H. corolla with staminode; I. anthers; J. fruit. K-O. Jacaranda jasminoides: K. flowering branch; L. calyx; M. flower; N. opened corolla showing stamens and staminode; O. fruit. Illustrations by Natanael Santos; A-E. from L.R. Silva 99; F and J. from L.R. Silva 262, G-I from J.B. Lima 161; K-O. from L.R. Silva 29. Figures K-O (Silva et al. 2016). 
According to Lohmann (2015) Jacaranda brasiliana is endemic to Brazil, distributed from the north to the southeast, in environments of Amazon Rainforest and Cerrado. Therefore, this work provides a new occurrence for this species in the Caatinga environments. In the studied Ecoregion is represented by few individuals. Flowers and fruits from November to January.

Species recognized by the presence of bipinnate leaves, leaflets small with base asymmetric cuneate, calyx deeply lobed, anthers 1thecate and woody capsules with margins broadly undulating.

16. Jacaranda jasminoides (Thunb.) Sandwith., Recueil Trav. Bot. Néerl. 34: 232. 1937. Figures 2O; 7K-O

Shrub erect ca. $3 \mathrm{~m}$; branchlets cylindrical, lightly striated, with lenticels, slightly pubescent, without interpetiolar glands. Prophylls of the axillary buds minute. Leaves pinnate at the base and bipinnate at the apex, 7-15-foliolate, petioles ca. $3 \mathrm{~cm}$, petiolules ca. $0.7 \mathrm{~cm}$, both pubescent, leaflets $3.5-6.5(-8) \times 2-4(-4.5) \mathrm{cm}$, discolorous, chartaceous, lanceolate or ovate, apex acute and obtuse, margins entire or serrated in young leaflets, base rounded to subcordate or cuneate in young leaflets, venation penninerved, adaxial surface slightly pubescent, abaxial surface tomentose, both with tector trichomes. Inflorescence a thyrse, peduncles ca. $4 \mathrm{~cm}$, pedicel ca. $1 \mathrm{~cm}$, both pubescent, bracts and bracteoles ca. $3 \mathrm{~mm}$. Calyx ca. $1 \times 0.5 \mathrm{~cm}$, green in the upper portion and vinaceous-magenta in the lower portion, cupulate, 5 -lobed, cuspidate, without rifts, pubescent, with glandular trichomes, without nectaries; corolla 6-7.5 × 1.5-2.5 cm, vinaceous-magenta with white nectar guide on the fauces, tubular-campanulate, slightly pubescent; stamens included, dorsal filaments ca. $2.3 \mathrm{~cm}$, ventral filaments ca. $2 \mathrm{~cm}$, anthers ca. $6 \mathrm{~mm}, 2$-thecate, glabrous, straight, connective not elongated, glabrous, staminode ca. $30 \mathrm{~mm}$, glandular-pubescent; nectariferous disk conspicuous, ovary ca. $3 \times 1 \mathrm{~mm}$, glabrous, sessile, ridged, style ca. $2.3 \mathrm{~cm}$, stigma ca. $2 \mathrm{~mm}$. Capsules 3-6.5 × ca. $5 \mathrm{~cm}$, ovate to obovate, compressed, smooth, coriaceous, margins flat, without wings. Seeds $0.8-1.5 \times$ ca. $2 \mathrm{~cm}$.

Material examined: BRAZIL. BAHIA: Euclides da Cunha, Sucupira do Galo, $10^{\circ} 21^{\prime} 01.1^{\prime \prime} \mathrm{S} 38^{\circ} 41^{\prime} 1.5^{\prime \prime} \mathrm{W}, 547 \mathrm{~m}$, 16.I.2014, fl.; fr., L.R. Silva et al. 268 (HUNEB); Jeremoabo, APA Serra Branca, Faz. Serra Branca, Estrada secundária principal próximo a entrada da caixa d'água, 0956'57"S 38²7'39"W, 555 m, 1.VI.2011, fl., L.R. Silva et al. 29 (HUNEB); APA Serra Branca, trilha da vaca morta, 0954'59.2"S $38^{\circ} 41^{\prime} 54.0^{\prime \prime} \mathrm{W}, 363$ m, 04.XI.2011, fl., L.R. Silva et al. 56 (HUNEB); Baixa da forra, $10^{\circ} 00^{\prime} 05.0^{\prime \prime} \mathrm{S} 38^{\circ} 23^{\prime} 39.6^{\prime \prime} \mathrm{W}, 564 \mathrm{~m}, 10 . \mathrm{V} .2013$, fl., L.R. Silva et al. 171 (HUNEB); Baixa dos Quelés, 0958'60"S 38 266'16"W, 488 m, 17.X.2009, fl.; fr., E. Melo et al. 6615 (HUEFS); Fazenda natureza, do Sr. Otávio Farias, ca. $20 \mathrm{~km}$ NO do município, caminho para o "Saco dos Cavalos", 1000'14"S 38 26'02"W, 427 m, 12.VIII.2005, fr., E.B. Miranda et al. 883 (HUEFS).

Jacaranda jasminoides is endemic to Brazil with records for the states of Alagoas, Bahia, Ceará, Paraíba, Pernambuco, Piauí, Rio Grande do Norte, Sergipe, Espírito Santo, Minas Gerais and Rio de Janeiro. It is distributed through environments of Caatinga, Cerrado and Atlantic Forest (Lohmann 2015). Species easily found by area. It flowers and fruiting the whole year.

This species is characterized by branches with pinnate leaves at base and bipinnate leaves at apex, leaflets with margins entire or serrate, flowers vinaceous-magenta with white nectar guides on the fauces, glandular-pubescent staminode ca. $30 \mathrm{~mm}$, and capsule ovate to obovate with flat margins.

\section{Lundia longa DC., Prodr. [A. P. de Candolle] 9: 180.}

1845. Figures 2P; 8A-E

Lianas; branchlets cylindrical, striated, with lenticels, glabrous, with interpetiolar glands. Prophylls of the axillary buds minute. Leaves 2-foliolate, petioles 2-4 cm, petiolules $1-2 \mathrm{~cm}$, tector trichomes sparse; leaflets 5-12 × 3-5.5 cm, discolorous, chartaceous, lanceolate to cordate, apex acuminate, margins entire, base subcordate, truncate or asymmetrical, venation penninerved, adaxial surface glabrescent, abaxial surface slightly pilose, with tector trichomes; tendrils simple, with tips not uncinate. Inflorescence a thyrse, peduncles $1-5.5 \mathrm{~cm}$, slightly pilose, pedicel ca. $1 \mathrm{~cm}$, glabrous or with tector trichomes sparse, bracts and bracteoles inconspicuous. Calyx ca. $9 \times 4 \mathrm{~cm}$, green to red, tubular, 5-lobed, irregular lobes, without rifts, slightly pilose, with tector trichomes, without nectaries; corolla ca. $6 \times 1 \mathrm{~cm}$, pink to red with yellow fauces, tubular to infundibuliform, pubescent; stamens exserted, dorsal filaments ca. $3 \mathrm{~cm}$, ventral filaments ca. $2.4 \mathrm{~cm}$, anthers ca. $6 \mathrm{~mm}, 2$-thecate, pilose, straight, connective not elongated, pilose, staminode ca. $5 \mathrm{~mm}$, glabrous; nectariferous disk absent, ovary ca. 3 $\times 1 \mathrm{~mm}$, pilose, sessile, no ridged, style ca. $5.3 \mathrm{~cm}$, stigma ca. $3 \mathrm{~mm}$. Fruits and seeds not seen.

Material examined: BRAZIL. BAHIA: Jeremoabo, APA Serra Branca, estrada para os Quelés, baixa da forra, depois do povoado Olhos d'água dos negros, 0959'37.8"S 38 23'45.8"W, 594 m, 19.XII.2012, fl., L.R. Silva et al. 87 (HUNEB); Estrada em direção aos Quelés, $10^{\circ} 00^{\prime} 09.2^{\prime \prime} \mathrm{S} 38^{\circ} 23^{\prime} 66.1^{\prime \prime} \mathrm{W}, 595 \mathrm{~m}, 22 . \mathrm{V} .2013$, fl., L.R. Silva et al. 220 (HUNEB); Estrada em direção ao povoado Quelés, 0959'13.2"S 38 24'57.3"W, 589 m, 22.V.2013, fl., L.R. Silva et al. 228 (HUNEB); Estada em direção ao povoado Quelés, $09^{\circ} 58^{\prime} 43.9^{\prime \prime} \mathrm{S} 38^{\circ} 25^{\prime} 50.9^{\prime \prime} \mathrm{W}, 510$ m, 19.XII.2013, fl., L.R. Silva et al. 242 (HUNEB); Faz. Natureza, de Sr. Otávio Farias, ca. 20 km NO do município caminho para o "Saco dos Cavalos", 1000'14"S 38²6'02"W, 427 m, 12.VIII.2005, fl., E.B. Miranda et al. 905 (HUEFS).

Lundia longa is endemic of the Brazil registered in wet sites in Atlantic Forest vegetation in eastern (Lohmann \& Taylor 2014). It is distributed by areas of Caatinga, Cerrado and Atlantic Forest, occurring in the states of Alagoas, Bahia, Ceará, Espírito Santo, Paraíba, Pernambuco, Sergipe, Minas Gerais, Rio de Janeiro, Rio Grande do Norte and São Paulo (Lohmann 2015). It flowers from May to December.

In the study area the species can be clearly differentiated from the others by lanceolate to cordate leaflets, flowers with pink to red corolla and yellow fauces, nectariferous disk absent, ovary, anthers and connective pilose.

18. Mansoa paganuccii M.M.Silva, Phytotaxa 258: 59. 2016. Figures 2Q; 8F-I

Lianas; branchlets cylindrical, striated, with lenticels, glabrous, without interpetiolar glands. Prophylls of the axillary buds minute. Leaves 2-3-foliolate, petioles $1.5-2 \mathrm{~cm}$, petiolules $0.2-0.8 \mathrm{~cm}$, both pubescent; leaflets $4.5-8 \times 2-3 \mathrm{~cm}$, discolorous, chartaceous, ovate to elliptic, apex acuminate or slightly emarginate, margins entire or irregularly serrate, base rounded, venation penninerved, both surfaces with tector trichomes restricted to the midrib; tendrils trifid, with tips not uncinate. Inflorescence a thyrse, peduncles ca. $2 \mathrm{~cm}$, glabrescent, 


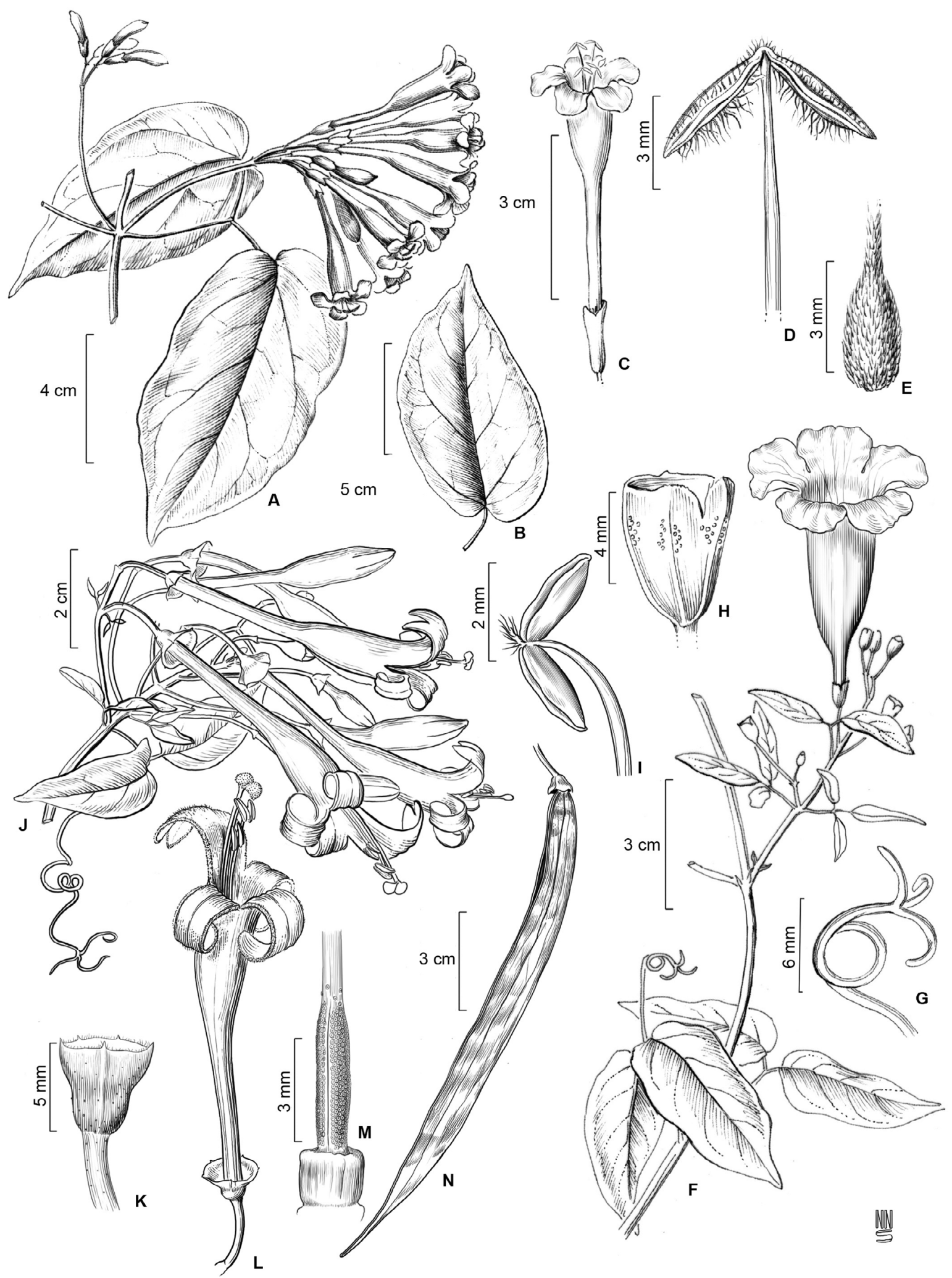

Figure 8. A-E. Lundia longa: A. flowering branch; B. leaflet; C. flower with stamens exserted; D. stamens; E. ovary. F-I. Mansoa paganuccii. F. flowering branch; G. tendril; H. calyx; I. stamens. J-N. Pyrostegia venusta: J. flowering branch; K. calyx; L. flower with stamens exserted; M. ovary; N. fruit. Illustrations by Natanael Santos; A-E. from A. S. Conceição 1777; F-I. from L.R. Silva 04; J-N. from D.D. Vieira 258. Figures A-I (Silva et al. 2016). 
pedicel ca. $1.3 \mathrm{~cm}$, glabrous, bracts and bracteoles inconspicuous. Calyx ca. $0.7 \times 0.5 \mathrm{~cm}$, green with magenta apex, campanulate, truncate or 5-mucronate, lobes inconspicuous, with rifts, glabrous, with nectaries; corolla 5-8 $\times 1.5-2 \mathrm{~cm}$, purple with purple fauces, infundibuliform, glabrous; stamens included, dorsal filaments ca. $2.3 \mathrm{~cm}$, ventral filaments ca. $1.8 \mathrm{~cm}$, anthers ca. $6 \mathrm{~mm}, 2$-thecate, glabrous, straight, connective elongated, pubescent, staminode ca. $6 \mathrm{~mm}$, glabrous; nectariferous disk conspicuous, ovary ca. $4 \times 1 \mathrm{~mm}$, lepidote, sessile, ridged, style ca. $2.3 \mathrm{~cm}$, stigma ca. $2 \mathrm{~mm}$. Capsules ca. $14 \times 1 \mathrm{~cm}$, linear, cylindrical, veins prominent, chartaceous, margins flat, without wings. Seeds ca. $1 \times 3.5 \mathrm{~cm}$.

Material examined: BRAZIL. BAHIA: Jeremoabo, APA Serra Branca, Caminho que vai da Estação Ecológica em direção ao povoado Quelés, 0956'36.1"S 38 27'12.1"W, 551 m, 23.IX.2009, fl., D.D. Vieira et al. 108 (HUNEB); Estrada que vai para os Quelés na entrada para Estação Ecológica Raso da Catarina, 0953'24.2"S 38³0'49.8'W, 550 m, 27.VII.2011, fl., L.R. Silva et al. 36 (HUNEB); Faz. Serra Branca, Trilha em direção ao tanque de dentro, 09²51'56.4"S 38³8'34.2"W, $484 \mathrm{~m}$, 04.XI.2010, fl., L.R. Silva et al. 04 (HUNEB); Faz. Serra Branca, trilha em direção ao Araça, 0954'12.2"S 38 37'55.3"W, 21.VIII.2008, fl., M.V.V. Romão et al. 262 (HUNEB); Paulo Afonso, Estação Ecológica Raso da Catarina, trilha sentido a mata da Pororoca, 09 48'91.0"S $38^{\circ} 28^{\prime} 81.8^{\prime \prime} \mathrm{W}, 543$ m, 01.I.2011, fl.; fr., L.R. Silva et al. 60 (HUNEB); Trilha em frente a casa do velho $09^{\circ} 42^{\prime} 08.1^{\prime \prime} \mathrm{S} 38^{\circ} 29^{\prime} 33.0^{\prime \prime} \mathrm{W}, 259 \mathrm{~m}$, 24.IX.2013, fl., L.R. Silva et al. 199 (HUNEB); Trilha do transecto, 09³9'50.7"S 38²8'01.4"W, 17.VIII.2010, fl., C.L.S.B. Correia et al. 178 (HUNEB); Santa Brígida, Fazenda do Sr. José Alves, 0944'15"S $38^{\circ} 17^{\prime} 60^{\prime \prime} \mathrm{W}, 407$ m, 28.XI.2009. fr., E. Melo, et al. 7289 (HUEFS).

Mansoa paganuccii is an endemic species of Brazil, with records for Caatinga vegetation in the northeast region, occurring in the states of Bahia, Ceará, Pernambuco, Piauí, Rio Grande do Norte and Minas Gerais (Silva-Castro \& Queiroz 2016, Lohmann 2015). In the Ecoregion is widely distributed throughout the area forming dense populations, mainly in the ESEC Raso da Catarina. Flowers and fruits from July to November.

This species is identified in the area for presents calyx truncate or 5-mucronate, purple corolla, stamens with an elongated and pubescent connective, cylindrical capsules with veins prominent. The absence of garlic odor is a good character used to differentiate it from other species of this genus.

\section{Pyrostegia venusta Miers, Proc. Roy. Hort. Soc. 3: 1863.} 188. Figures 2R; 8J-N

Lianas; branchlets hexagonal, striated, without lenticels, glabrous, without interpetiolar glands. Prophylls of the axillary buds minute. Leaves 2-foliolate, petioles $1.5-2 \mathrm{~cm}$, petiolules ca. $1 \mathrm{~cm}$, both pubescent; leaflets ca. $6 \times 2.5 \mathrm{~cm}$, concolorous, chartaceous, ovate, apex acuminate, margins entire, base rounded, venation penninerved, both surfaces glabrous, with few tector trichomes restricted to the midrib; tendrils trifid, with tips not uncinate. Inflorescence a corymb, peduncles ca. $2.5 \mathrm{~cm}$, pedicel $1-2 \mathrm{~cm}$, both glabrous, bracts and bracteoles ca. $9 \mathrm{~mm}$. Calyx ca. $0.6 \times 0.8 \mathrm{~cm}$, green, widely campanulate, 5-lobed, lobes inconspicuous, without rifts, glabrous, with tectores trichomes in the apex, without nectaries; corolla 6-7 $\times 1-1.5 \mathrm{~cm}$, orange, without nectar guides, tubular-infundibuliform, glabrous; stamens exserted, dorsal filaments ca. $3 \mathrm{~cm}$, ventral filaments ca. $2 \mathrm{~cm}$, anthers ca. 8 $\mathrm{mm}, 2$-thecate, glabrous, straight, connective not elongated, glabrous, staminode ca. $2 \mathrm{~mm}$, glabrous; nectariferous disk conspicuous, ovary ca. $5 \times 1 \mathrm{~mm}$, lepidote, sessile, ridged, style ca. $6 \mathrm{~cm}$, stigma ca. $3 \mathrm{~mm}$. Capsules ca. $16 \times 1 \mathrm{~cm}$, linear, compressed, smooth, coriaceous, margins flat, without wings. Seeds ca. $3.5 \times 1 \mathrm{~cm}$.

Material examined: BRAZIL. BAHIA: Canudos, Estação Biológica de Canudos, Toca velha saco 1 das Araras, 0957'20"S 38 59'98"W, 413 m, 26.IV.2012, fl.; fr., D.D. Vieira et al. 258 (HUNEB); Jeremoabo, APA Serra Branca, estrada sentido ao povoado Quelés, 09 58'73.5"S 38 26'73.3"W, 532 m, 22.X.2013, fl., L.R. Silva et al. 229 (HUNEB); Trilha sentido povoado Quelés, $10^{\circ} 00^{\prime} 46.8^{\prime \prime} \mathrm{S} 38^{\circ} 23^{\prime} 21.7^{\prime \prime} \mathrm{W}$, 565 m, 22.X.2013, fl.; fr., L.R. Silva et al. 216 (HUNEB).

The species widely and commonly found in dry to humid lowland forests has records for Mexico to Argentina (Lohmann \& Taylor 2014). It is widely distributed in Brazil occurring from the north to the south, in environments of Amazon Rainforest, Caatinga, Cerrado, Atlantic Forest, Pampa and Pantanal (Lohmann 2015). In the study area it is often found. Flowers and fruits from June to October.

Pyrostegia venusta can be easily recognized by branchlets hexagonal, trifid tendrils, with tips not uncinate, widely campanulate calyx, orange corolla and exserted stamens.

\section{Tabebuia aurea (Manso) Benth. \& Hook f. ex Moore,}

Trans. Linn. Soc. London, Bot. 4: 423. 1895. Figure 9A-E

Tree ca. $6 \mathrm{~m}$; branchlets cylindrical, without streaking, without lenticels, glabrous. Without interpetiolar glands. Prophylls of the axillary buds minute. Leaves digitate, (3)5-foliolate, petioles 3-10 $\mathrm{cm}$, petiolules $1-5 \mathrm{~cm}$, glabrous or lepidote; leaflets $10-18 \times 3-4 \mathrm{~cm}$, concolorous, coriaceus, lanceolate or eliptic, apex cuneate or rounded, margins entire, base truncate or cuneate, occasionally cordate, venation penninerved, adaxial and abaxial surfaces lepidote. Inflorescence a thyrse, peduncles ca. 1-3 cm, pedicel ca. $1-1.5 \mathrm{~cm}$, both lepidote, bracts and bracteoles inconspicuous. Calyx $1-2 \times$ ca. $0.8 \mathrm{~cm}$, green, tubular, bilabiate, lepidote, without rifts, without nectaries; corolla 5-9 $\times$ ca. $2 \mathrm{~cm}$, yellow with red or brown nectar guides on the fauces, infundibuliform, glabrous; stamens included, dorsal filaments ca. 1.7 $\mathrm{cm}$, ventral filaments ca. $1.2 \mathrm{~cm}$, anthers ca. $8 \mathrm{~mm}$, 2-thecate, glabrous, straight, connective not elongated, glabrous, staminode ca. $8 \mathrm{~mm}$, glabrous; nectariferous disk conspicuous, ovary ca. $4 \times 1 \mathrm{~mm}$, lepidote, sessile, not ridged, style ca. $3.5 \mathrm{~cm}$, stigma ca. $2 \mathrm{~mm}$. Capsules 9-17× 1.4-2 cm, linear to oblong, rounded, smooth, coriaceous, margins flat, without wings. Seeds $1.1-1.4 \times 0.8-0.9 \mathrm{~cm}$.

Material examined: BRAZIL. BAHIA: Canudos, Estação Ecológica de Canudos, área da base Elyseu, 0956'S 3858'W, 22.X.2004, fl., F.H.M. Silva \& L.C.L. Lima 513 (HUEFS); Parque Estadual de Canudos-PEC, próximo ao alto da faucela, vizinho a casa de cheiroso, 0954'42.7"S 3904'30.7"W, 389 m, 10.X.2008, fl., C.L.S.B. Correria et al.46 (HUNEB); Jeremoabo, APA Serra Branca, Faz. Serra Branca, 0957'23.8"S 38²'23.4"W, 01.XI.2008, fl., M.V.V. Romão et al. 412 (HUNEB); APA Serra Branca, Faz. Serra Branca, margens do rio Vaza Barris, 0957'23.8"S 38 42'23.4"W, 23.X.2013, fl., L.R. Silva et al. 234 (HUNEB); Arredores da cidade, s/c, 08.IV.1983, fl., M.C. Ferreira 500 (HRB); Estrada em direção ao povoado Os Brancos, $10^{\circ} 05^{\prime} 89.7^{\prime \prime} \mathrm{S} 38^{\circ} 23^{\prime} 38.6^{\prime \prime} \mathrm{W}, 264$ m, 23.X.2013, fl., L.R. Silva et al. 238 (HUNEB); Estrada de chão para Canudos, 10 $0^{\circ} 6^{\prime} 10^{\prime \prime} \mathrm{S} 38^{\circ} 25^{\prime} 48^{\prime \prime} \mathrm{W}, 533$ m, 30.VI.2007, fl., M.M. Silva-Castro et al. 1238 (HUEFS); Rodelas, ca. 
Silva, L.R. et al.
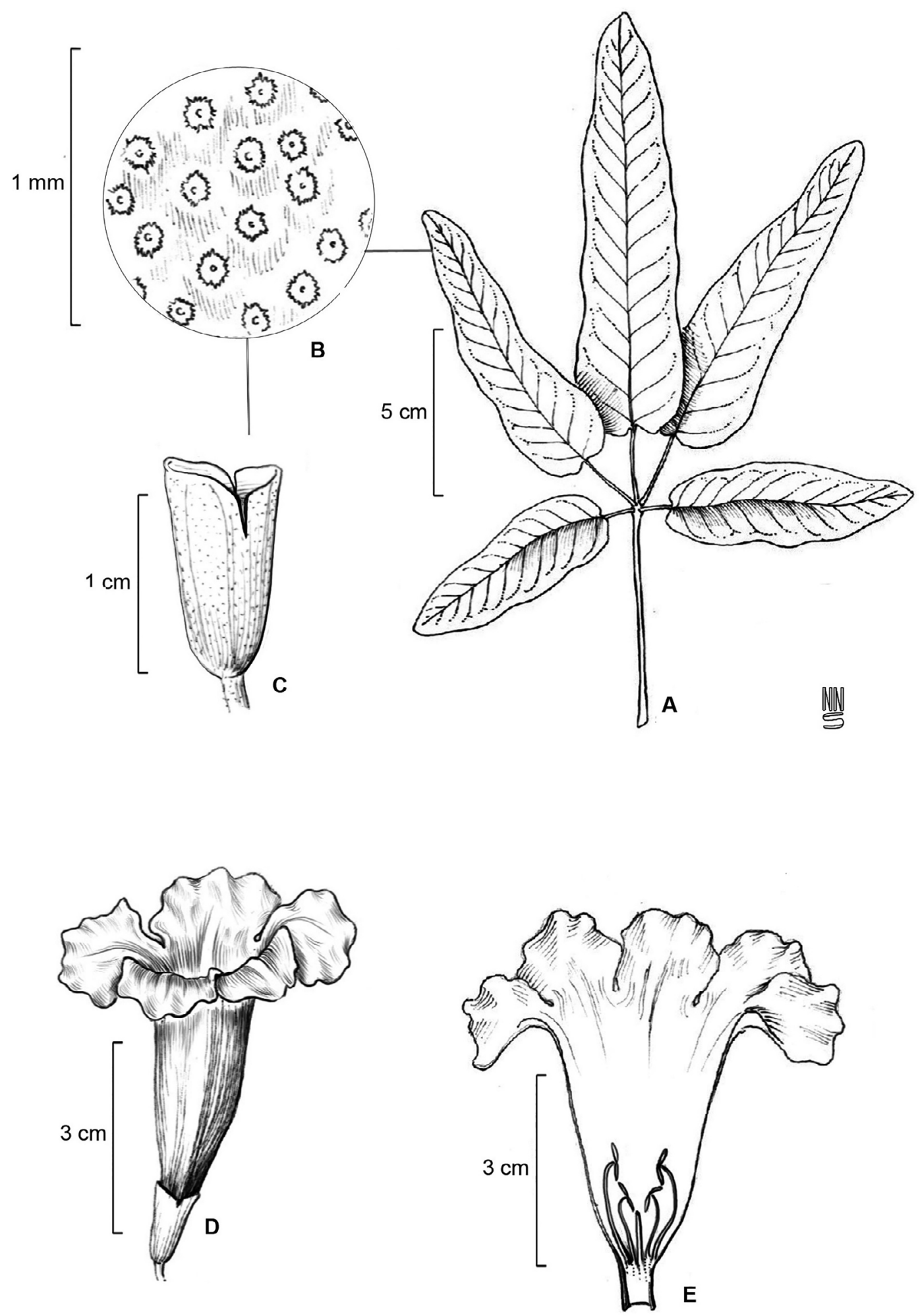

Figure 9. A-E. Tabebuia aurea: A. leaf; B. indument of leaflet and calyx; C. calyx; D. flower; E. Opened corolla showing stamens and staminode. Illustrations by Natanael Santos; A-E. from M.V.V. Romão 412. (Silva et al. 2016). 
$14,5 \mathrm{~km}$ da cidade, beira do rio seco com açude, $08^{\circ} 52^{\prime} 33^{\prime \prime} \mathrm{S} 38^{\circ} 54^{\prime} 34^{\prime \prime} \mathrm{W}$ 345 m, 30.X.2009, fl.; fr., E. Melo et al. 6987 (HUEFS); Santa Brígida, fazenda do Sr. José Alves, 0944'07"S 3809'21"'W, 323 m, 28.XI.2009, fl., E. Melo et al. 7273 (HUEFS).

A typical species of Brazilian cerrado, it also occurs in seasonal forests of Argentina, western Bolivia and Southeast Suriname (Gentry 1992b). In Brazil it is widely distributed, occurring from the north to the south, in environments of Amazon Rainforest, Caatinga, Cerrado, Atlantic Forest and Pantanal (Lohmann 2015). In the study area the species is common, being much used as ornamental. Flowers and fruit in September until January with peak of flowering between September and October.

Tabebuia aurea is recognized by digitate leaves with lepidote indumentum, flowers with bilabiate calyx, lepidote and the corolla is yellow with red or brown nectar guides on the fauces.

\section{Supplementary Material}

The following online material is available for this article:

Appendix 1. List of additional material examined

\section{Acknowledgements}

Thanks to the Fundação de Amparo à Pesquisa do Estado da Bahia (FAPESB, PET \# 0023/2007) and to the Conselho Nacional de Desenvolvimento Científico e Tecnológico (CNPq Proc. \# 552589/2011$0)$ for financial support. The first author thanks the Coordenação de Aperfeiçoamento de Pessoal de Nível Superior (CAPES) by scholarship, the curators and technicians of the herbaria that were visited for their readiness during the consultation of the collections and Natanael Santos for the botanical illustrations.

\section{Author Contributions}

Luiza Regina Silva: Contribution to the acquisition of data; analysis and interpretation of data; work of writing and conception and design work.

Milene Maria da Silva Castro: Contribution to the interpretation of data and critical review adding intellectual content.

Adilva de Souza Conceição: Contribution to the acquisition of data; analysis and interpretation of data; work of writing; critical review adding intellectual content and conception and design work.

\section{Conflicts of interest}

The authors declare that they have no conflict of interest related to the publication of this manuscript.

\section{References}

AMORIM, V.O. \& BAUTISTA, H.P. 2016. Asteraceae da Ecorregião Raso da Catarina, Bahia, Brasil. Rodriguésia 67(3):785-794.

APG (Angiosperm Phylogeny Group). 2016. An update of the Angiosperm Phylogeny Group classification for the orders and families of flowering plants: APG IV. Bot. J. Linn. Soc. 181:1-20.
BRITO, A.F.S. 2012. Levantamento florístico e anatomia foliar de Acacieae e Ingeae (Leguminosae: Mimosoideae) na Apa Serra Branca/Raso da Catarina, Bahia, Brasil. Dissertação de mestrado, Universidade do Estado da Bahia, Paulo Afonso.

CORREIA, C.L.S.B. \& CONCEIÇÃO, A.S. 2017a. The genus Chamaecrista Moench in a fragment of the Ecological Station Raso da Catarina, Bahia, Brazil. Biota Neotrop. 17(2):1-16. e20160225. http://dx.doi. org/10.1590/1676-0611-BN-2016-0225 (last access on 13/10/2017)

CORREIA, C.L.S.B. \& CONCEIÇÃO, A.S. 2017b. The genus Senna Mill. (Leguminosae: Caesalpinioideae) in a fragment of the Ecological Station Raso da Catarina, Bahia, Brazil. Acta Sci. Biol. Sci. 39(3):357-372.

DOURADO, D.A.O., CONCEIÇÃO, A.S. \& SANTOS-SILVA, J. 2013. O gênero Mimosa L. (Leguminosae: Mimosoideae) na APA Serra Branca/ Raso da Catarina, Bahia, Brasil. Biota Neotrop. 13(4):225-240. http://www. biotaneotropica.org.br/v13n4/pt/abstract?inventory+bn01713042013 (last access in 13/10/2017)

ESPÍRITO SANTO, F.S., SILVA-CASTRO, M.M. \& RAPINI, A. 2012. Handroanthus grandiflorus (Bignoniaceae), a new species from the semiarid region of Brazil. Phytotaxa 48(1):1-6.

ESPÍRITO SANTO, F.S., SILVA-CASTRO, M.M. \& RAPINI, A. 2013. Flora da Bahia: Bignoniaceae 2 - Aliança Tabebuia (Bignoniaceae). Sitientibus ser. Ci. Biol. 13:1-38.

FOSBERG, F.R. \& SACHET, M.H. 1965. Manual for tropical herbaria. Utrecht, Netherlands.

GENTRY, A.H. 1973. Flora of Panamá: Bignoniaceae. Ann. Mo. Bot. Gard. 60(3):781-977.

GENTRY, A.H. 1980. Bignoniaceae, Part I, Tribes Crescentieae and Tourretieae. Flora Neotropica 25(1):1-131.

GENTRY, A.H. 1982. Bignoniaceae. Flora de Colombia. Instituto de Ciencias Naturales. Universidad Nacional de Colombia, Bogota.

GENTRY, A.H. 1986. Species richness and floristic composition of Chocó plant communities. Caldasia 15:71-91.

GENTRY, A.H. 1992a. A synopsis of Bignoniaceae ethnobotany and economic botany. Ann. Mo. Bot. Gard. 79(1):53-64.

GENTRY, A.H. 1992b. Bignoniaceae Part II. Tribe Tecomeae. Flora Neotropica 25(2):1-362.

GIULIETTI, A.M., HARLEY, R.M., QUEIROZ, L.P, BARBOSA, M.R.V., BOCAGE, A.L.N, \& FIGUEIREDO, M.A. 2002. Espécies endêmicas da Caatinga. In Vegetação e Flora da Caatinga (E. Sampaio, A.M. Giulietti, J. Virgínio, \& C. Gamarra-Rojas, eds.). Associação Plantas do Nordeste/ NIP, Recife, p.103-119.

GONÇALVES, E.G. \& LORENZI, H. 2011. Morfologia vegetal: organografia e dicionário ilustrado de morfologia das plantas vasculares. 2 ed. Instituto Plantarum, São Paulo.

GROSE, S.O. \& OLMSTEAD, R.G. 2007. Taxonomic Revisions in the Polyphyletic Genus Tabebuia s.1. (Bignoniaceae). Syst. Bot. 32(3):660-670.

HARRIS, J.G. \& HARRIS M.W. 2001. Plant identification terminology: an illustrated glossary. Spring Lake Publishing, Spring Lake.

LEAL, I.R., SILVA, J.M.C., TABARELLI, M. \& LACHER JR, T.E. 2005. Mudando o curso da conservação da biodiversidade na Caatinga do Nordeste do Brasil. Megadiversidade 1(1):139-146.

LIMA, J.B. \& CONCEIÇÃO, A.S. 2016. Malvoideae Burnett (Malvaceae) in the Environmental Protection Area Serra Branca, Raso da Catarina, Jeremoabo, Bahia, Brazil. Biota Neotrop. 16(4):e20160187. http://dx.doi org/10.1590/1676-0611-BN-2016-0187 (last access in 13/10/2017).

LIMA, J.B. 2015. A família Malvaceae Sensu Lato na Ecorregião Raso da Catarina, Bahia, Brasil. Dissertação de mestrado, Universidade do Estado da Bahia, Paulo Afonso.

LOHMANN, L.G. \& PIRANI, J.R. 1996. Tecomeae (Bignoniaceae) da Cadeia do Espinhaço, Minas Gerais e Bahia, Brasil. Acta Bot. Bras. 10(1):103-137.

LOHMANN, L.G. \& TAYLOR, C.M. 2014. A new generic classification of tribe Bignonieae (Bignoniaceae). Ann. Mo. Bot. Gard. 99(3):348-489. 
LOHMANN, L.G. \& ULLOA ULLOA, C. 2007. Bignoniaceae. In iPlants Prototype Checklist. Available at: www.iplants.org (last aceess in 13/10/2017).

LOHMANN, L.G. 2004. Bignoniaceae. In Flowering Plants of the Neotropics (N. Smith, S.A. Mori, A. Henderson, D.W. Stevenson, \& S.V. Heald, eds.). Princeton University Press, New Jersey, p.51-53.

LOHMANN, L.G. 2015. Bignoniaceae. In Lista de Espécies da Flora do Brasil. Jardim Botânico do Rio de Janeiro. Available at: http://floradobrasil.jbrj.gov. br/ jabot/floradobrasil/FB112532 (last access in 13/10/2017).

LOPES, A.A. DE S. 2012. Diversidade de Euphorbiaceae nas caatingas arenosas da APA Serra Branca, Jeremoabo, Bahia, Brasil. Dissertação de mestrado, Universidade do Estado da Bahia, Paulo Afonso.

LORENZI, H. 2008. Árvores Brasileiras: manual de identificação e cultivo de plantas arbóreas do Brasil. Instituto Plantarum, Nova Odessa.

LORENZI, H. 2013. Plantas para jardim no Brasil: herbáceas, arbustivas e trepadeiras. Instituto Plantarum, Nova Odessa.

MELO, J.I., M., ALVES. I. DE M., SOUSA, R.T.M., BARBOSA, L.M.A. \& ANDRADE, W.M. 2010. Verbenaceae Sensu Lato em um trecho da ESEC Raso da Catarina, Bahia, Brasil. Rev. Caatinga 23:41-47.

MELO, J.I.M. \& ANDRADE, W.M. 2007. Boraginaceae Sensu Lato Juss. em uma área de Caatinga da ESEC Raso da Catarina, BA, Brasil. Acta Bot. Bras. 2:369-378.

MORI, S.A., SILVA, A.M., LISBOA, A. \& CORADIN, L. 1989. Manual de manejo de herbário fanerogâmico. Ed. 2. Centro de pesquisas do Cacau, Ilhéus.

OLMSTEAD, R.G., ZJHRA, M. L., LOHMANN, L.G., GROSE, S.O. \& ECKERT, A.J. 2009. A molecular phylogeny and classification of Bignoniaceae. Am. J. Bot. 96(9):1731-1743.

RADFORD, A.E., DICKISON, W.C., MASSEY, J.R. \& BELL, R. 1974. Vascular plant systematics. Harper \& Row publishers, New York.

RIZZINI, C.M., AGAREZ, F.V., ANDRADE, L.H.C. \& AZEVEDO, A.P. 1997. A família Bignoniaceae na APA de Maricá, Rio de Janeiro, Brasil. Acta Bot. Bras. 11(2):153-163.

SAMPAIO, E.V.S.B. 1995. Overview of the Brazilian caatinga. In Seasonally dry tropical forests (S.H. Bullock, H.A. Mooney \& E. Medina, eds.). Cambridge University Press, Cambridge, p. 34-63.

SANTOS, J.V. 2016. A família Malpighiaceae Juss. na Ecorregião Raso da Catarina, Bahia, Brasil. Dissertação de mestrado, Universidade do Estado da Bahia, Paulo Afonso.

SANTOS, J.V., NUNES, T.S. \& CONCEIÇÃO, A.S. 2016. A família Passifloraceae na APA Serra Branca/Raso da Catarina, Jeremoabo, Bahia, Brasil. Biotemas 29(1): 11-23.

SANTOS, L.L., SANTOS, L.L., ALVES, A.S.A., OLIVEIRA, L.S.D. \& SALES, M.F. 2013. Bignoniaceae Juss. no Parque Nacional Vale do Catimbau, Pernambuco. Rodriguésia 64(3):479-494
SCUDELLER, V.V. 2004. Bignoniaceae Juss. no Parque Nacional da Serra da Canastra - Minas Gerais, Brasil. Iheringia 59(1):59-73.

SILVA, D. DA C. 2014. Apocynaceae s.1. no Raso da Cararina, Bahia, Brasil, com ênfase nas suas Unidades de Conservação: riqueza e análise de similaridade. Dissertação de mestrado, Universidade do Estado da Bahia, Paulo Afonso.

SILVA, L.R., SILVA-CASTRO, M.M. \& CONCEIÇÃO, A.S. 2016. The family Bignoniaceae in the Environmental Protection Area Serra Branca, Raso da Catarina, Jeremoabo, Bahia, Brazil. Acta Sci. Biol. Sci. 38(4):395-409.

SILVA, M.M. \& QUEIROZ, L.P. 2003. A família Bignoniaceae na região de Catolés, Chapada Diamantina, Bahia, Brasil. Sitientibus ser. Ci. Biol. $3(1 / 2): 3-21$

SILVA-CASTRO, M.M. \& QUEIROZ, L.P. 2016. Five new species of Mansoa DC. (Bignoniaceae) from South America. Phytotaxa 258(1):49-62.

SILVA-CASTRO, M.M., COSTA, C.R. A. \& BRITO, R.F. 2007. Flora da Bahia - Bignoniaceae 1: Jacaranda Jussieu. Sitientibus ser. Ci. Biol. 7(1):15-31.

SPANGLER, R.E. \& OLMSTEAD, R.G. 1999. Phylogenetic analysis of Bignoniaceae based on the cpDNA gene sequences rbcL and ndhF. Ann. Mo. Bot. Gard. 86(1):33-46.

SZABO, A.V., ROCHA, A.C.S., TOSATO, J.A. C. \& BARROSO, W. 2007. Área de proteção ambiental (APA) Serra Branca Raso da Catarina. In As Caatingas: debates sobre a ecorregião do Raso da Catarina (J. Marques, org.). Fonte Viva, Paulo Afonso, p.21-40.

THIERS, B. 2018 [continuously updated]. Index Herbariorum: a global directory of public herbaria and associated staff. In New York Botanical Garden's Virtual Herbarium. Available at: http://sweetgum.nybg.org/science/ih/ (last access in 16/06/2018)

TRICART, J. 1972. The landforms of the humid tropics, forests and savannas. Geographies for Advanced Study. Longman, London.

VARJÃO, R.R., JARDIM, J.G. \& CONCEICÃO, A.S. 2013. Rubiaceae Juss. de caatinga na APA Serra Branca/Raso da Catarina, Bahia, Brasil. Biota Neotrop. 13(2): http://www.biotaneotropica.org.br/v13n2/en/abstract?inv entory+bn00313022013 (last access in 13/10/2017)

VELLOSO, A.L., SAMPAIO, E.V.S.B. \& PAREYN, F.G.C. 2002. Ecorregiões propostas para o bioma Caatinga. Associação Plantas do Nordeste, Recife.

VIEIRA, D.D., CONCEIÇÃO, A.S., MELO, J.I.M \& STAPF, M.N.S. 2013. A família Boraginaceae sensu lato na APA Serra Branca/Raso da Catarina, Bahia, Brasil. Rodriguésia 64(1):151-168.

VIEIRA, D.D., MELO, J.I.M \& CONCEIÇÃO, A.S. 2015. Boraginales Juss. ex Bercht. \& J.Presl in the Ecoregion Raso da Catarina, Bahia, Brazil. Biota Neotrop. 15(3): http://dx.doi.org/10.1590/1676-0611-BN-2014-0201 (last access in 13/10/2017)

WOODSON, R.E., SCHERY, R.W. \& GENTRY, A.H. 1973. Flora of Panama. Part IX. Family 172. Bignoniaceae. Ann. Mo. Bot. Gard. 60(3):781-977.

Received: $16 / 10 / 2017$

Revised: $18 / 06 / 2018$

Accepted: 23/08/2018

Published online: 17/09/2018 